\title{
Kostka-Foulkes Polynomials Cyclage Graphs and Charge Statistic for the Root System $C_{n}$
}

CÉDRIC LECOUVEY

lecouvey@math.unicaen.fr

Received November 11, 2003; Revised March 24, 2004; Accepted April 18, 2004

Abstract. We establish a Morris type recurrence formula for the root system $C_{n}$. Next we introduce cyclage graphs for the corresponding Kashiwara-Nakashima's tableaux and use them to define a charge statistic. Finally we conjecture that this charge may be used to compute the Kostka-Foulkes polynomials for type $C_{n}$.

Keywords: crystal graphs, cyclage graphs, Kostka-Foulkes polynomials

\section{Introduction}

The multiplicity $K_{\lambda, \mu}$ of the weight $\mu$ in the irreducible finite dimensional representation $V(\lambda)$ of the simple Lie algebra $g$ can be written in terms of the ordinary Kostant's partition function $\mathcal{P}$ defined from the equality:

$$
\prod_{\alpha \text { positive root }} \frac{1}{\left(1-x^{\alpha}\right)}=\sum_{\beta} \mathcal{P}(\beta) x^{\beta}
$$

where $\beta$ runs on the set of nonnegative integral combinations of positive roots of $g$. Thus $\mathcal{P}(\beta)$ is the number of ways the weight $\beta$ can be expressed as a sum of positive roots. Then we have

$$
K_{\lambda, \mu}=\sum_{\sigma \in W}(-1)^{l(\sigma)} \mathcal{P}(\sigma(\lambda+\rho)-(\mu+\rho))
$$

where $W$ is the Weyl group of $g$.

There exists a $q$-analogue $K_{\lambda, \mu}(q)$ of $K_{\lambda, \mu}$ obtained by substituting the ordinary Kostant's partition function $\mathcal{P}$ by its $q$-analogue $\mathcal{P}_{q}$ satisfying

$$
\prod_{\alpha \text { positive root }} \frac{1}{\left(1-q x^{\alpha}\right)}=\sum_{\beta} \mathcal{P}_{q}(\beta) x^{\beta} \text {. }
$$

So we have

$$
K_{\lambda, \mu}(q)=\sum_{\sigma \in W}(-1)^{l(\sigma)} \mathcal{P}_{q}(\sigma(\lambda+\rho)-(\mu+\rho)) .
$$


As shown by Lusztig [17] $K_{\lambda, \mu}(q)$ is a polynomial in $q$ with non negative integer coefficients. These polynomials naturally appear in the classical theory of Hall-Littlewood polynomials. They coincide with the Kostka-Foulkes polynomials that is, with the entries of the transition matrix between the basis of Hall-Littelwood polynomials and the basis of Schur functions [18]. Many interpretations of the Kostka-Foulkes polynomials exist. For example, they appear in the filtrations of weight spaces by the kernels of powers of a regular nilpotent element, and degree in harmonic polynomials $[1,2,6]$. We recover them in the expansion of the Hall Littlewood polynomials in terms of the affine Hecke algebra (see [19]).

For type $A_{n-1}$ the positivity of the Kostka-Foulkes Polynomials can also be proved by a purely combinatorial method. Recall that for any partitions $\lambda$ and $\mu$ with $n$ parts the number of semi-standard tableaux of shape $\lambda$ and weight $\mu$ is equal to the multiplicity of the weight $\mu$ in the finite dimensional irreducible module of $U_{q}\left(s l_{n}\right)$ with highest weight $\lambda$. In [14] Lascoux and Schützenberger have introduced a beautiful statistic $\mathrm{ch}_{A}$ on standard tableaux called the charge and, by using Morris recurrence formula, have proved the equality

$$
K_{\lambda, \mu}(q)=\sum_{T \in S T(\mu)_{\lambda}} q^{\mathrm{ch}_{A}(T)}
$$

where $S T(\mu)_{\lambda}$ is the set of semi-standard tableaux of shape $\lambda$ and weight $\mu$. Set $\mathcal{A}_{n}=$ $\{1<\cdots<n\}$. The charge may be defined by endowing $S T(\mu)$ the set of semi-standard tableaux of weight $\mu$ with a structure of graph defined from Lascoux-Schützenberger's plactic monoid. Recall that the plactic monoid is the quotient set of $\mathcal{A}_{n}^{*}$ the free monoid on $\mathcal{A}_{n}$ by the Knuth relations

$$
a b x \equiv\left\{\begin{array}{ll}
b a x & \text { if } a<x \leq b \\
a x b & \text { if } x \leq a<b
\end{array} .\right.
$$

For any tableau $T$ we denote by $\mathrm{w}(T)$ the column reading of $T$ that is, the word obtained by reading the columns of $T$ from right to left and from top to bottom. The cyclage graph structure on $S T(\mu)$ can be defined as follows. We draw an arrow $T \rightarrow T^{\prime}$ between the two tableaux $T$ and $T^{\prime}$ of $S T(\mu)$, if and only if there exists $u$ in $\mathcal{A}_{n}^{*}$ and $x \neq 1 \in \mathcal{A}_{n}$ such that $\mathrm{w}(T) \equiv x u$ and $\mathrm{w}\left(T^{\prime}\right) \equiv u x$. Then we say that $T^{\prime}$ is a cocyclage of $T$. The essential tool to define this graph structure is the insertion algorithm for the semi-standard tableaux. The cyclage graph $S T(\mu)$ contains a unique row tableau $L_{\mu}$ which can not be obtained as the cocyclage of another tableau of $S T(\mu)$. Let $T_{\mu}$ be the unique semi-standard tableau of shape $\mu$ belonging to $S T(\mu)$. Then there is no cocyclage of $T_{\mu}$. For any $T \in S T(\mu)$ all the paths joining $L_{\mu}$ to $T$ have the same length. This length is called the cocharge of $T$ and denoted $\operatorname{coch}_{A}(T)$. Similarly, all the paths joining $T$ to $T_{\mu}$ have the same length called the charge of $T$. The maximal value of $\mathrm{ch}_{A}$ is $\|\mu\|=\mathrm{ch}_{A}\left(L_{\lambda}\right)=\sum_{i}(i-1) \mu_{i}$. Moreover the charge and the cocharge satisfy the equality $\operatorname{ch}_{A}(T)=\|\mu\|-\operatorname{coch}_{A}(T)$ for any $T \in S T(\mu)$.

The initial cocyclage of the tableau $T$ of reading $\mathrm{w}(T)=x u$ with $x \neq 1$ is obtained by inserting $x$ in the sub-tableau of $T$ of reading $u$. Every tableau $T \in S T(\mu)$ can be related to $T_{\mu}$ by a sequence of initial cocyclages. So it is enough to consider initial cocyclages to define $\mathrm{ch}_{A}$. 
The charge of $T$ can also be defined directly from $\mathrm{w}(T)$ when $\mu$ is a dominant weight. Moreover it can be characterized in terms of the geometry of the crystal graph $B(\lambda)$ associated to $V(\lambda)[12]$.

In this article we restrict ourselves to the root system $C_{n}$. Our aim is two folds. First we establish Morris type recurrence formula for type $C_{n}$ and use it to give explicit formulae for Kostka-Foulkes polynomials when $\lambda$ is a row partition or a column partition of height 2 . Next we introduce a cyclage graph structure and a notion of charge for type $C_{n}$. For any dominant weight $\lambda$, let $V(\lambda)$ be the finite dimensional irreducible $U_{q}\left(s p_{2 n}\right)$-module with highest weight $\lambda$. In Kashiwara and Nakashima [11] have given a combinatorial description of $B(\lambda)$ the crystal graph of $V(\lambda)$ in terms of symplectic tableaux analogous to the semistandard tableaux for type $C_{n}$. From the plactic monoid and the insertion algorithm described in [15] it is natural to try to obtain cyclage graphs for symplectic tableaux. Nevertheless the situation is more complex than for type $A_{n-1}$. First we have to restrict the possible cocyclage operations to the initial cocyclage to avoid loops in our cyclage graphs. Moreover if we use the complete insertion algorithm for type $C_{n}$, the number of boxes of the cocyclage of a tableau $T$ may be strictly less than that of $T$ due to the contraction relation in the plactic monoid. The cyclage graphs obtained by this mean seem to be not relevant to define a charge related to the Kostka-Foulkes polynomials. To overcome this problem we will execute the insertion algorithm without this contraction relation and consider that the symplectic tableaux are filled by letters of the totally ordered alphabet

$$
\mathcal{C}_{n}=\{\bar{n}<\cdots<\overline{1}<1<\cdots<n\}
$$

which can be naturally embedded in the infinite alphabet

$$
\mathcal{C}_{\infty}=\{\cdots<\bar{n}<\cdots<\overline{1}<1<\cdots<n<\cdots\} .
$$

Our convention for the alphabet $\mathcal{C}_{n}$ is not identical to that of [11] to dispose of a natural infinite extension of $\mathcal{C}_{n}$. Denote by $\mathbf{S T}(n)$ the set of symplectic tableaux defined on $\mathcal{C}_{n}$. If $T \in \mathbf{S T}(n)$ the initial cocyclage (without contraction) of $T$ does not belong to $\mathbf{S T}(n)$ in general but belongs to $\mathbf{S T}(n+1)$. So it is natural to consider the cyclage graph structure of $\mathbf{S T}=\cup_{n \geq 1} \mathbf{S T}(n)$.

Let $\mu$ be a dominant weight for the root system $C_{n}$. With our convention $\mu$ may be identified with the partition $\left(\mu_{\bar{n}}, \ldots, \mu_{\overline{1}}\right)$. We are going to endow $\mathbf{S T}(\mu)$ the subset of $\mathbf{S T}$ containing the symplectic tableaux $T$ such that for any $m \geq 1$, the number of letters $\bar{k}$ in $T$ minus the number of letters $k$ is $\mu_{\bar{k}}$ if $k \leq n, 0$ otherwise with a structure of cyclage graph. This structure is more complex than for type $A_{n}$. In particular $\mathbf{S T}(\mu)$ decomposes into connected components. These components can be isomorphic and do not necessarily contain a row tableau. We define on $\mathbf{S T}(\mu)$ a charge statistic $\mathrm{ch}_{n}$. Many computations allows us to conjecture that an analogue to (1) exists for type $C_{n}$ with the charge $\mathrm{ch}_{n}$. However it seems to be impossible to derive it from our Morris type recurrence formula.

In Section 1 we recall the Background on Kostka-Foulkes polynomials and crystal basis theory that we need in the sequel. We also summarize the basic properties of the insertion algorithm introduced in [15]. Section 2 is devoted to the Morris type recurrence formula for 
type $C_{n}$ and its applications. In Section 3 we define the cyclage graph structure on $\mathbf{S T}(\mu)$ and give some of its properties. Finally we introduced $\mathrm{ch}_{n}$ in Section 4 and conjecture that it permits to compute the Kostka-Foulkes polynomials for type $C_{n}$.

\section{Background}

\subsection{Kostka-Foulkes polynomials for type $C_{n}$}

We choose to label the Dynkin diagram of $s p_{2 n}$ by

$$
\stackrel{0}{\circ} \Rightarrow \stackrel{1}{\circ}-\stackrel{2}{\circ}-\stackrel{3}{\circ}-\stackrel{4}{\circ}-\cdots \stackrel{n-1}{\circ} \text {. }
$$

The weight lattice $P_{n}$ of $C_{n}$ can be identified with $\mathbb{Z}^{n}$ equipped with the orthonormal basis $\varepsilon_{\bar{i}}, i=1, \ldots, n$. We take for the simple roots

$$
\alpha_{0}=2 \varepsilon_{\overline{1}} \quad \text { and } \quad \alpha_{i}=\varepsilon_{\overline{i+1}}-\varepsilon_{\bar{i}}, i=1, \ldots, n-1 .
$$

Then the set of positive roots of $s p_{2 n}$ is

$$
R_{n}^{+}=\left\{\varepsilon_{\bar{i}}^{\overline{-}}-\varepsilon_{\bar{j}}^{\bar{j}}, \varepsilon_{\bar{i}}^{\bar{*}}+\varepsilon_{\bar{j}}^{-} \text {with } 1 \leq j<i \leq n\right\} \cup\left\{2 \varepsilon_{i}^{-} \text {with } 1 \leq i \leq n\right\} .
$$

Denote by $P_{n}^{+}$the set of dominant weights of $s p_{2 n}$. Write $\Lambda_{0}, \ldots, \Lambda_{n-1}$ for the fundamentals weights. Then we have $\Lambda_{i}=\varepsilon_{\bar{n}}+\cdots+\varepsilon_{\overline{i+1}}, 0 \leq i \leq n-1$. Consider $\lambda \in P_{n}^{+}$and set $\lambda=\sum_{i=0}^{n-1} \hat{\lambda}_{i} \Lambda_{i}$ with $\hat{\lambda}_{i} \in \mathbb{N}$. The dominant weight $\lambda$ is characterized by the partition $\left(\lambda_{\bar{n}}, \ldots, \lambda_{\overline{1}}\right)$ where $\lambda_{\bar{i}}=\hat{\lambda}_{0}+\cdots+\hat{\lambda}_{i-1}, i=1, \ldots, n$. In the sequel we will identify $\lambda$ and $\left(\lambda_{\bar{n}}, \ldots, \lambda_{\overline{1}}\right)$ by setting $\lambda=\left(\lambda_{\bar{n}}, \ldots, \lambda_{\overline{1}}\right)$. Then $\lambda=\lambda_{\overline{1}} \varepsilon_{\overline{1}}+\cdots+\lambda_{\bar{n}} \varepsilon_{\bar{n}}$ that is, the $\lambda_{i}$ 's are the coordinates of $\lambda$ on the basis $\left(\varepsilon_{\bar{n}}, \ldots, \varepsilon_{\overline{1}}\right)$. Let $\rho$ be the half sum of positive roots. We have $\rho=(n, n-1, \ldots, 1)$. For any $\lambda \in P_{n}^{+}$, set $|\lambda|=\lambda_{\bar{n}}+\cdots+\lambda_{\overline{1}}$.

The Weyl group $W_{n}$ of $s p_{2 n}$ can be regarded as the sub group of the permutation group of $\mathcal{C}_{n}=\{\bar{n}, \ldots, \overline{2}, \overline{1}, 1,2, \ldots, n\}$ generated by $s_{i}=(i, i+1)(\bar{i}, \overline{i+1}), i=1, \ldots, n-1$ and $s_{0}=(1, \overline{1})$ where for $a, b \in \mathcal{C}_{n}$ with $a \neq b,(a, b)$ is the simple transposition which switches $a$ and $b$. Note that any $\sigma \in W_{n}$ verifies $\sigma(\bar{i})=\overline{\sigma(i)}$ for $i \in\{1, \ldots, n\}$. We denote by $l$ the length function corresponding to the set of generators $s_{i}, i=0, \ldots n-1$.

The action of $\sigma \in W_{n}$ on $\beta=\left(\beta_{\bar{n}}, \ldots, \beta_{\overline{1}}\right) \in P_{n}$ is given by

$$
\sigma \cdot\left(\beta_{\bar{n}}, \ldots, \beta_{\overline{1}}\right)=\left(\beta_{\bar{n}}^{\sigma}, \ldots, \beta_{\overline{1}}^{\sigma}\right)
$$

where $\beta_{\bar{i}}^{\sigma}=\beta_{\sigma(\bar{i})}$ if $\sigma(\bar{i}) \in\{\overline{1}, \ldots, \bar{n}\}$ and $\beta_{\bar{i}}^{\sigma}=-\beta_{\sigma(i)}$ otherwise.

Let $Q_{n}^{+}$be the set of nonnegative integral linear combinations of positive roots. For any $\beta=\left(\beta_{\bar{n}}, \ldots, \beta_{\overline{1}}\right) \in P_{n}$ we set $x^{\beta}=x_{n}^{\beta_{\bar{n}}} \ldots x_{1}^{\beta_{\overline{1}}}$ where $x_{1}, \ldots, x_{n}$ are fixed indeterminates. The $q$-analogue $\mathcal{P}_{q}$ of the Kostant function partition is defined by

$$
\prod_{\alpha \in R_{n}^{+}} \frac{1}{1-q x^{\alpha}}=\sum_{\beta \in Q_{n}^{+}} \mathcal{P}_{q}(\beta) x^{\beta} \quad \text { and } \quad \mathcal{P}_{q}(\beta)=0 \quad \text { if } \quad \beta \notin Q_{n}^{+} .
$$


Definition 2.1.1 Let $\lambda, \mu \in P_{n}^{+}$. The Kostka-Foulkes polynomial $K_{\lambda, \mu}(q)$ is defined by

$$
K_{\lambda, \mu}(q)=\sum_{\sigma \in W_{n}}(-1)^{l(\sigma)} \mathcal{P}_{q}(\sigma(\lambda+\rho)-(\mu+\rho))
$$

Let $\beta \in P_{n}$. We set

$$
a_{\beta}=\sum_{\sigma \in W_{n}}(-1)^{l(\sigma)}\left(\sigma \cdot x^{\beta}\right)
$$

where $\sigma \cdot x^{\mu}=x^{\sigma(\mu)}$. The Schur function $s_{\beta}$ is defined by

$$
s_{\beta}=\frac{a_{\beta+\rho}}{a_{\rho}} .
$$

When $\lambda \in P_{n}^{+}, s_{\lambda}$ is the Weyl character of $V(\lambda)$ the finite dimensional irreducible $U_{q}\left(s p_{2 n}\right)$ module with highest weight $\lambda$. For any $\sigma \in W_{n}$, the dot action of $\sigma$ on $\beta \in P_{n}$ is defined by $\sigma \circ \beta=\sigma \cdot(\beta+\rho)-\rho$. We have the following straightening law for the Schur functions. For any $\beta \in P_{n}, s_{\beta}=0$ or there exists a unique $\lambda \in P_{n}^{+}$such that $s_{\beta}=(-1)^{l(\sigma)} s_{\lambda}$ with $\sigma \in W_{n}$ and $\lambda=\sigma \circ \beta$. Set $\mathbb{K}=\mathbb{Z}\left[q, q^{-1}\right]$ and write $\mathbb{K}\left[P_{n}\right]$ for the $\mathbb{K}$ module generated by the $x^{\beta}, \beta \in P_{n}$. Set $\mathbb{K}\left[P_{n}\right]^{W_{n}}=\left\{f \in \mathbb{K}\left[P_{n}\right], \sigma \cdot f=f\right.$ for any $\left.\sigma \in W_{n}\right\}$. Then $\left\{s_{\lambda}\right\}$ is a basis of $\mathbb{K}\left[P_{n}\right]^{W_{n}}$.

To each positive root $\alpha$, we associate the raising operator $R_{\alpha}: P_{n} \rightarrow P_{n}$ defined by

$$
R_{\alpha}(\beta)=\alpha+\beta
$$

Given $\alpha_{1}, \ldots, \alpha_{p}$ positive roots and $\beta \in P_{n}$, we set $\left(R_{\alpha_{1}} \ldots R_{\alpha_{p}}\right) s_{\beta}=s_{R_{\alpha_{1}} \ldots R_{\alpha_{p}}(\beta)}$. Composing the action of raising operators on Schur function should be avoided in general. For example $\left(R_{\alpha_{1}} R_{\alpha_{2}}\right)\left(s_{\beta}\right)$ is not necessarily equal to $\left(R_{\alpha_{1}}\right)\left(R_{\alpha_{2}} s_{\beta}\right)$ (see example p 360 in [19]). For all $\beta \in P_{n}$, we define the Hall-Littelwood polynomial $Q_{\beta}$ by

$$
Q_{\beta}=\left(\prod_{\alpha \in R_{n}^{+}} \frac{1}{1-q R_{\alpha}}\right) s_{\beta}
$$

where $\frac{1}{1-q R_{\alpha}}=\sum_{k=0}^{+\infty} q^{k} R_{\alpha}^{k}$.

Theorem 2.1.2 [19] For any $\lambda, \mu \in P_{n}^{+}, K_{\lambda, \mu}(q)$ is the coefficient of $s_{\lambda}$ in $Q_{\mu}$ that is,

$$
Q_{\mu}=\sum_{\lambda \in P_{n}^{+}} K_{\lambda, \mu}(q) s_{\lambda}
$$

When $n=1$, the root system $C_{1}$ can be regarded as the root system $A_{1}$ and the KostkaFoulkes polynomial $K_{\lambda, \mu}(q)$ where $\lambda$ and $\mu$ are partitions of length 1 satisfies

$$
K_{\lambda, \mu}(q)=q^{(|\lambda|-|\mu|) / 2}
$$


Lemma 2.1.3 Consider $\lambda, \mu \in P_{n}^{+}$such that $\lambda_{\bar{n}}=\mu_{\bar{n}}$ and write $\lambda^{\prime}=\left(\lambda_{\overline{n-1}}, \ldots, \lambda_{\overline{1}}\right)$, $\mu^{\prime}=\left(\mu_{\overline{n-1}}, \ldots, \mu_{\overline{1}}\right)$. Then $K_{\lambda, \mu}(q)=K_{\lambda^{\prime}, \mu^{\prime}}(q)$.

Proof: $K_{\lambda, \mu}(q)$ is the coefficient of $s_{\lambda}$ in

$$
\begin{aligned}
& \prod_{\alpha \in R_{n}^{+}}\left(\frac{1}{1-q R_{\alpha}}\right) s_{\mu} \\
& \quad=\left(\prod_{1 \leq j \leq n} \frac{1}{1-q R_{2 \varepsilon_{j}}} \prod_{1 \leq j<i \leq n} \frac{1}{1-q R_{\varepsilon_{\bar{i}}-\varepsilon_{\bar{j}}}} \prod_{1 \leq j<i \leq n} \frac{1}{1-q R_{\varepsilon_{\bar{i}}+\varepsilon_{\bar{j}}}}\right) s_{\mu}
\end{aligned}
$$

but since $\lambda_{\bar{n}}=\mu_{\bar{n}}$ none of the raising operators involving $\varepsilon_{\bar{n}}$ contribue to $K_{\lambda, \mu}(q)$. Thus $K_{\lambda, \mu}(q)=K_{\lambda^{\prime}, \mu^{\prime}}(q)$, the coefficient of $s_{\lambda^{\prime}}$ in

$$
\prod_{1 \leq j \leq n-1}\left(\frac{1}{1-q R_{2 \varepsilon_{\bar{j}}}} \prod_{1 \leq j<i \leq n-1} \frac{1}{1-q R_{\varepsilon_{\bar{i}}-\varepsilon_{\bar{j}}}} \prod_{1 \leq j<i \leq n-1} \frac{1}{1-q R_{\varepsilon_{\bar{i}}+\varepsilon_{\bar{j}}}}\right) s_{\mu^{\prime}} .
$$

\subsection{Crystal Graphs for Type $C_{n}$}

Recall that crystal graphs for the $U_{q}\left(s p_{2 n}\right)$-modules are oriented colored graphs with colors $i \in\{0, \ldots, n-1\}$. An arrow $a \stackrel{i}{\rightarrow} b$ means that $\tilde{f}_{i}(a)=b$ and $\tilde{e}_{i}(b)=a$ where $\tilde{e}_{i}$ and $\tilde{f}_{i}$ are the crystal graph operators (for a review of crystal bases and crystal graphs see [10]). A vertex $v^{0} \in B$ satisfying $\tilde{e}_{i}\left(v^{0}\right)=0$ for any $i \in\{0, \ldots, n-1\}$ is called a highest weight vertex. The decomposition of $V$ into its irreducible components is reflected into the decomposition of $B$ into its connected components (see (6) below). Each connected component of $B$ contains a unique highest weight vertex. The crystals graphs of two isomorphic irreducible components are isomorphic as oriented colored graphs. The action of $\tilde{e}_{i}$ and $\tilde{f}_{i}$ on $B \otimes B^{\prime}=\left\{b \otimes b^{\prime}\right.$; $\left.b \in B, b^{\prime} \in B^{\prime}\right\}$ is given by:

$$
\tilde{f}_{i}(u \otimes v)= \begin{cases}\tilde{f}_{i}(u) \otimes v & \text { if } \varphi_{i}(u)>\varepsilon_{i}(v) \\ u \otimes \tilde{f}_{i}(v) & \text { if } \varphi_{i}(u) \leq \varepsilon_{i}(v)\end{cases}
$$

and

$$
\tilde{e_{i}}(u \otimes v)= \begin{cases}u \otimes \tilde{e_{i}}(v) & \text { if } \varphi_{i}(u)<\varepsilon_{i}(v) \\ \tilde{e_{i}}(u) \otimes v & \text { if } \varphi_{i}(u) \geq \varepsilon_{i}(v)\end{cases}
$$


where $\varepsilon_{i}(u)=\max \left\{k ; \tilde{e}_{i}^{k}(u) \neq 0\right\}$ and $\varphi_{i}(u)=\max \left\{k ; \tilde{f}_{i}^{k}(u) \neq 0\right\}$. The weight of the vertex $u$ is defined by $\operatorname{wt}(u)=\sum_{i=0}^{n-1}\left(\varphi_{i}(u)-\varepsilon_{i}(u)\right) \Lambda_{i}$.

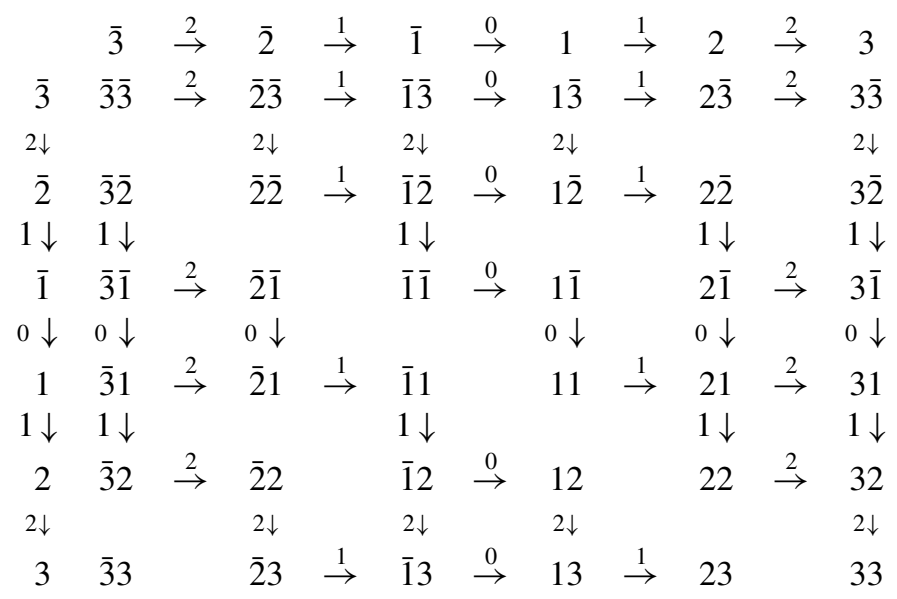

The 3 connected components of $B\left(\Lambda_{2}\right)^{\otimes 2}$ for $n=3$.

The following lemma is a straightforward consequence of (4) and (5).

Lemma 2.2.1 Let $u \otimes v \in B \otimes B^{\prime} u \otimes v$ is a highest weight vertex of $B \otimes B^{\prime}$ if and only if for any $i \in\{0, \ldots, n-1\} \tilde{e}_{i}(u)=0$ (i.e. $u$ is of highest weight) and $\varepsilon_{i}(v) \leq \varphi_{i}(u)$.

The Weyl group $W_{n}$ acts on $B$ by:

$$
\begin{aligned}
& s_{i}(u)=\left(\tilde{f_{i}}\right)^{\varphi_{i}(u)-\varepsilon_{i}(u)}(u) \quad \text { if } \varphi_{i}(u)-\varepsilon_{i}(u) \geq 0, \\
& s_{i}(u)=\left(\tilde{\tilde{e}_{i}}\right)^{\varepsilon_{i}(u)-\varphi_{i}(u)}(u) \quad \text { if } \varphi_{i}(u)-\varepsilon_{i}(u)<0 .
\end{aligned}
$$

We have the equality $\operatorname{wt}(\sigma(u))=\sigma(\operatorname{wt}(u))$ for any $\sigma \in W_{n}$ and $u \in B$. For any $\lambda \in P_{n}^{+}$, we denote by $B(\lambda)$ the crystal graph of $V(\lambda)$.

According to (2) we have

$$
B\left(\Lambda_{n-1}\right): \bar{n} \stackrel{n-1}{\rightarrow} \overline{n-1} \stackrel{n-2}{\rightarrow} \cdots \rightarrow \overline{2} \stackrel{1}{\rightarrow} \overline{1} \stackrel{0}{\rightarrow} 1 \stackrel{1}{\rightarrow} 2 \ldots \stackrel{n-2}{\rightarrow} n-1 \stackrel{n-1}{\rightarrow} n .
$$

Kashiwara-Nakashima's combinatorial description of the crystal graphs $B(\lambda)$ is based on the notion of symplectic tableaux analogous for type $C_{n}$ to semi-standard tableaux.

We defined a total order on $\mathcal{C}_{n}$ by setting

$$
\mathcal{C}_{n}=\{\bar{n}<\cdots<\overline{1}<1<\cdots<n\} .
$$

For any letter $x \in \mathcal{C}_{n}$ we set $\overline{\bar{x}}=x$. Note that our convention for labelling the crystal graph of the vector representation are not those used by Kashiwara and Nakashima. To obtain the 
original description of $B(\lambda)$ from that used in the sequel it suffices to change each letter $k \in\{1, \ldots, n\}$ of $\mathcal{C}_{n}$ into $\overline{n-k+1}$ and each letter $\bar{k} \in\{\overline{1}, \ldots, \bar{n}\}$ into $n-k+1$. The interest of this change of convention will appear in Sections 4 and 5.

We identify the vertices of the crystal graph $G_{n}=\oplus_{l} B\left(\Lambda_{n-1}\right)^{\otimes l}$ with the words on $\mathcal{C}_{n}$. For any $w \in G_{n}$ we have $\operatorname{wt}(w)=d_{\bar{n}} \varepsilon_{\bar{n}}+d_{\overline{n-1}} \varepsilon_{n-1} \cdots+d_{\overline{1}} \varepsilon_{\overline{1}}$ where for any $i=1, \ldots, n$ $d_{\bar{i}}$ is the number of letters $\bar{i}$ of $w$ minus the number of its letters $i$. Using Formulas (4) and (5) we obtain a simple rule to compute the action of $\tilde{e}_{i}, \tilde{f}_{i}$ or $s_{i} i \in\{0, \ldots, n-1\}$ on $w \in G_{n}$ that we will use in Section 4. Consider the subword $w_{i}$ of $w$ containing only the letters $\overline{i+1}, \bar{i}, i, i+1$ if $i \neq 0$ and the letters $\overline{1}, 1$ otherwise. Then encode in $w_{i}$ each letter $\overline{i+1}$ or $i$ by the symbol + and each letter $\bar{i}$ or $i+1$ by the symbol - . Because $\tilde{e}_{i}( \pm)=\tilde{f}_{i}( \pm)=0$ in $B\left(\Lambda_{n-1}\right) \otimes B\left(\Lambda_{n-1}\right)$ the factors of type \pm may be ignored in $w_{i}$. So we obtain a subword $w_{i}^{(1)}$ in which we can ignore all the factors \pm to construct a new subword $w_{i}^{(2)}$ etc... Finally we obtain a subword $\rho(w)$ of $w$ of type

$$
\rho(w)=-^{r}+{ }^{s}
$$

Then we have the

\section{Note 2.2.2}

- When $r>0, \tilde{e}_{i}(w)$ is obtained by changing the rightmost symbol - of $\rho(w)$ into its corresponding symbol + (i.e. $i+1$ into $i$ and $\bar{i}$ into $\overline{i+1}$ if $i \neq 0,1$ into $\overline{1}$ otherwise) the others letters of $w$ being unchanged. When $r=0, \tilde{e}_{i}(w)=0$.

- When $s>0, \tilde{f}_{i}(w)$ is obtained by changing the leftmost symbol + of $\rho(w)$ into their corresponding symbols - (i.e. $i$ into $i+1$ and $\overline{i+1}$ into $\bar{i}$ if $i \neq 0, \overline{1}$ into 1 otherwise) the others letters of $w$ being unchanged. When $s=0, \tilde{f}_{i}(w)=0$.

- When $r \geq s, s_{i}(w)$ is obtained by changing the $r-s$ rightmost symbols - of $\rho(w)$ into its corresponding symbol + . When $r<s, s_{i}(w)$ is obtained by changing the $s-r$ leftmost symbols + of $\rho(w)$ into their corresponding symbols - .

In the sequel we will need the following definitions and notation:

A column on $\mathcal{C}_{n}$ is a Young diagram $C$ of column shape filled from top to bottom by increasing letters of $\mathcal{C}_{n}$.

The height $h(C)$ of a column $C$ is the number of its letters.

$\mathbf{C}(n, h)$ is the set of columns of height $h$ on $\mathcal{C}_{n}$ i.e. with letters in $\mathcal{C}_{n}$.

The reading of $C \in \mathbf{C}(n, h)$ is the word $w(C)$ of $\mathcal{C}_{n}^{*}$ obtained by reading its letters from top to bottom.

The column $C$ contains the pair $(z, \bar{z})$ when $C$ contains the unbarred letter $z \geq 1$ and the barred letter $\bar{z} \leq \overline{1}$.

Let $C_{1}$ and $C_{2}$ be two columns. We will write $C_{1} \leq C_{2}$ when $h\left(C_{1}\right) \geq h\left(C_{2}\right)$ and the rows of the tableau $C_{1} C_{2}$ weakly increase. 
Definition 2.2.3 Let $C$ be a column on $\mathcal{C}_{n}$ and $I_{C}=\left\{z_{1}<\cdots<z_{r}\right\}$ the set of unbarred letters $z$ such that the pair $(z, \bar{z})$ occurs in $C$. The column $C$ is $n$-admissible when there exists a set of unbarred letters $J_{C}=\left\{t_{1}<\cdots<t_{r}\right\} \subset \mathcal{C}_{n}$ such that:

- $t_{1}$ is the lowest letter of $\mathcal{C}_{n}$ satisfying: $t_{1}>z_{1}, t_{1} \notin C$ and $\overline{t_{1}} \notin C$,

- for $i=2, \ldots, r, t_{i}$ is the lowest letter of $\mathcal{C}_{n}$ satisfying: $t_{i}>\max \left(t_{i-1,}, z_{i}\right), t_{i} \notin C$ and $\overline{t_{i}} \notin C$.

In this case we write:

- $r C$ for the column obtained from $C$ by changing $z_{i}$ into $t_{i}$ for each letter $z_{i} \in I_{C}$,

- $l C$ for the column obtained from $C$ by changing $\bar{z}_{i}$ into $\bar{t}_{i}$ for each letter $z_{i} \in I_{C}$.

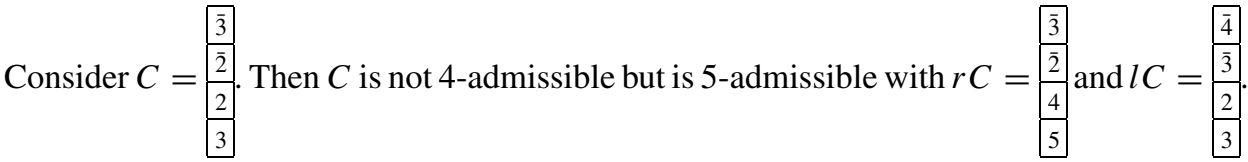

As usually, we associate to each partition $\lambda=\left(\lambda_{\bar{n}}, \ldots, \lambda_{\overline{1}}\right)$ the Young diagram $Y(\lambda)$ whose $i$-th row has length $\lambda \overline{n-i+1}$. By definition, a $n$-symplectic tableau $T$ of shape $\lambda$ is a filling of $Y(\lambda)$ by letters of $\mathcal{C}_{n}$ satisfying the following conditions:

- the columns $C_{i}$ of $T=C_{1} \cdots C_{s}$ are $n$-admissible,

- for $i=1, \ldots, s-1: r C_{i} \leq l C_{i+1}$.

The set of $n$-symplectic tableaux will be denoted $\mathbf{S T}(n)$.

If $T=C_{1} C_{2} \cdots C_{r} \in \mathbf{S T}(n)$, the reading of $T$ is the word $w(T)=w\left(C_{r}\right) \cdots w\left(C_{2}\right) w\left(C_{1}\right)$.

From [11] we deduce the

\section{Theorem 2.2.4}

(i) The vertices of $B\left(\Lambda_{p}\right) p=0, \ldots, n-1$ are in one-to-one correspondence with the readings of $n$-admissible columns of height $n-p$.

(ii) The vertices of $B(\lambda)$ are in one-to-one correspondence with the readings of the $n$ symplectic tableaux of shape $\lambda$.

More precisely Kashiwara and Nakashima realize $B(\lambda)$ into a tensor power $B\left(\Lambda_{n-1}\right)^{\otimes l}$. Given $p=0, \ldots, n-1, B\left(\Lambda_{p}\right)$ can then be identified with the connected component of $G_{n}$ whose highest weight vertex is $b_{\bar{p}}=\bar{n}(\overline{n-1}) \cdots \overline{p+1}$. In this identification, the vertices of $B\left(\Lambda_{p}\right)$ are the readings of the admissible columns of height $n-p$. If $\lambda=\sum_{p=0}^{n-1} \hat{\lambda}_{p} \Lambda_{p}$, $B(\lambda)$ is identified with the connected component whose highest weight vertex is $b_{\lambda}=$ $b_{\bar{n}}^{\otimes \hat{\lambda}_{n}} \cdots \otimes b_{\overline{1}}^{\otimes \hat{\lambda}_{1}} b_{0}^{\otimes \hat{\lambda}_{0}}$.

By identifying $U_{q}\left(s p_{2(n-1)}\right)$ with the sub-algebra of $U_{q}\left(s p_{2 n}\right)$ generated by the Chevalley's generators $e_{i}, f_{i}$ and $t_{i}, i=0, \ldots, n-1$, we endow $B(\lambda)$ with a structure of crystal graph for type $C_{n-1}$. The decomposition of $B(\lambda)$ into its $U_{q}\left(s p_{2(n-1)}\right)$-connected components is obtained by erasing all the arrows of color $n-1$. 


\subsection{Insertion scheme for symplectic tableaux}

In [15] we have introduced an insertion scheme for symplectic tableaux analogous for type $C_{n}$ to the bumping algorithm on Young tableaux. Now we are going to summarize the properties of this scheme that we shall need in Section 4.

Consider first a letter $x$ and a column $C$. The insertion of the letter $x$ in the $n$-admissible column $C$ is denoted $x \rightarrow C$. If $x$ is strictly greater than the greatest letter of $C$ then $x \rightarrow C$ is the column obtained by adding a box containing $x$ on bottom of $C$, that is, $x \rightarrow C=\frac{C}{x}$. Now suppose that $x$ is less than the greatest letter of $C$. Then $x \rightarrow C$ is a symplectic tableau of two columns defined recursively as follows:

if $C=$ contains only one column then $x \rightarrow a=x \mid a$

if $C=\frac{a}{b}$ contains two letters,

1. $x \rightarrow \frac{a}{b}=\frac{a}{x} b$ if $a<x \leq b$ and $b \neq \bar{a}$,

2. $x \rightarrow \frac{a}{b}=\frac{x}{b}|a|$ if $x \leq a<b$ and $b \neq \bar{x}$,

3. $x \rightarrow \frac{\overrightarrow{\bar{b}}}{b}=\frac{\overline{\overline{b+1}} b+1}{x}$ if $a=\bar{b}$ and $\bar{b} \leq x \leq b$,

4. $\bar{b} \rightarrow \frac{\bar{a}}{\bar{b}}=\overline{\overline{b-1}} \mid \overrightarrow{b-1}$ if $x=\bar{b}$ and $\bar{b}<a<b$.

Consider a $n$-admissible column $C$ of height $k \geq 3$ and suppose we have defined our insertion for the $n$-admissible columns of height $<k$. If $w(C)=a_{1}$, dots, $a_{k-1} a_{k}$ and

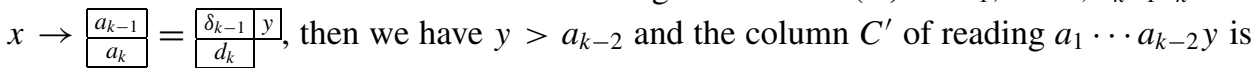
$n$-admissible. Write

$$
\delta_{k-1} \rightarrow C^{\prime}=\begin{array}{|c|c|}
\hline d_{1} & z \\
\hline \cdot & \\
\hline \cdot & \\
\hline d_{k-1} & \\
\cline { 1 - 1 } &
\end{array}
$$

and set

$$
x \rightarrow C=\begin{array}{|c|c|}
\hline d_{1} & \mathrm{Z} \\
\hline \cdot & \\
\hline \cdot & \\
\cline { 1 - 1 } d_{k-1} & \\
\hline d_{k} & \\
\hline
\end{array}
$$


This can be pictured by

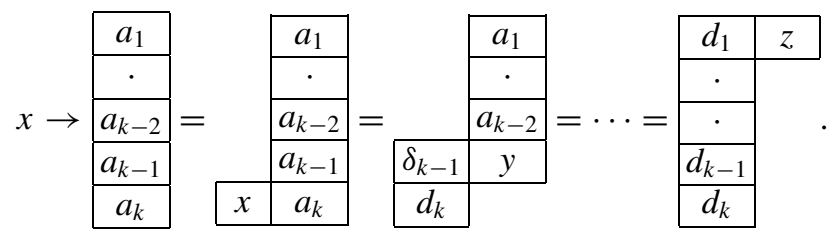

During each step we apply one of the transformations 1 to 4 above. We have proved in [15] that $x \rightarrow C$ is then a $n$-symplectic tableau with two columns respectively of height $h(C)$ and 1.

Example 2.3.1 Suppose $n=5$.

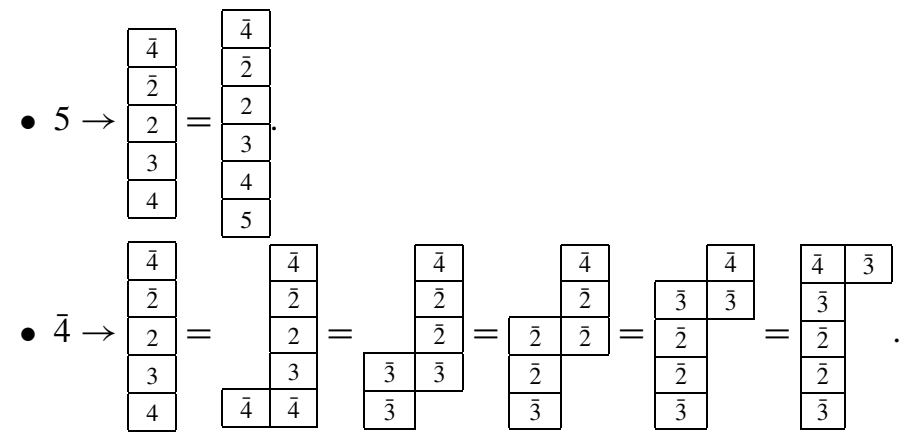

\section{Remarks}

(i) If $C$ is $n$-admissible and $x \rightarrow C$ is a column, then this column is $(n+1)$-admissible but not necessarily $n$-admissible.

(ii) From transformations 1 to 4 we obtain the plactic relations

$$
\begin{aligned}
a b x= & \left\{\begin{array}{l}
b a x \quad \text { if } a<x \leq b \text { and } \quad b \neq \bar{a} \\
a x b \text { if } x \leq a<b \text { and } b \neq \bar{x}
\end{array}\right. \text { and } \\
& \begin{cases}\bar{b} b x=(b+1)(\overline{b+1}) x \quad \text { if } \bar{b} \leq x \leq b \\
a b \bar{b}=a(\overline{b-1})(b-1) \text { if } \bar{b}<a<b\end{cases}
\end{aligned}
$$

introduced in [15]. These relations are not sufficient to define a plactic monoid for type $C_{n}$. We need a contraction relation which permits to obtain a $n$-admissible column from a non $n$-admissible one. Let $C^{\prime}=\frac{C}{\frac{C}{x}}$ be a non $n$-admissible column on $\mathcal{C}_{n}$ such that $C$ is $n$-admissible and $x$ a letter. In this case we can prove that there exists an unbarred letter $z$ 
maximal such that the pair $(z, \bar{z})$ occurs in $C^{\prime}$ and

$$
\operatorname{card}\left\{t \in \mathcal{C}_{n},|t| \geq z\right\}>n-z+1 \text {. }
$$

Write $D$ for the column obtained by erasing the pair $(z, \bar{z})$ in $C^{\prime}$. Then $D$ is $n$-admissible and the contraction relation is defined by

$$
w\left(C^{\prime}\right) \equiv_{n} w(D)
$$

In fact this last relation is not needed to define the cocyclage in Section 4.

Definition 2.3.2 The congruence obtained by identifying the words of $\mathcal{C}_{n}^{*}$ which are equal up to relations $(8)$ is denoted $\equiv$.

(iii): When $x \rightarrow C=C^{\prime} \mid y$ is a tableau of two columns we have

$$
h(C)=h\left(C^{\prime}\right) \text { and } \quad C^{\prime} \leq C .
$$

Now we can define the insertion $x \rightarrow T$ of the letter $x$ in the $n$-symplectic tableau $T$. Write $T=C_{1} \cdots C_{r}$ where $C_{i}, i=1, \ldots, r$ are the $n$-admissible columns of $T$. If $x$ is strictly greater to the greatest letter of $C_{1}$ then $x \rightarrow T$ is the tableau obtained by adding a box containing $x$ on bottom of $C_{1}$. Then $x \rightarrow T$ belongs to $\mathbf{S T}(n+1)$ but not to $\mathbf{S T}(n)$ in general since its first column may be non $n$-admissible. Otherwise write $x \rightarrow C=C_{1}^{\prime} y$ where $C_{1}^{\prime}$ is an admissible column of height $h\left(C_{1}\right)$ and $y$ a letter. Then $x \rightarrow T=C_{1}^{\prime}\left(y \rightarrow C_{2} \cdots C_{r}\right)$ that is, $x \rightarrow T$ is the juxtaposition of $C_{1}^{\prime}$ with the tableau obtained by inserting $y$ in the tableau $C_{2} \cdots C_{r}$. In this case $x \rightarrow T$ is a $n$-symplectic tableau [15].

Example 2.3.3 Suppose $n=3$. Then

$$
2 \rightarrow\left(1 \rightarrow \begin{array}{|l|l|l}
\hline \overline{1} & 1 & 2 \\
\hline 1 & 2 & \\
\cline { 1 - 2 } 3 & \multicolumn{1}{|l}{}
\end{array}\right)=\begin{array}{|l|l|l|l|}
\hline \overline{2} & 1 & 2 & 2 \\
\hline 1 & 2 & \multicolumn{3}{|c}{} \\
\cline { 1 - 3 } 2 & 3 & \multicolumn{3}{|c}{} \\
\cline { 1 - 3 }
\end{array}
$$

\section{Remarks}

(i) The insertion scheme described above do not suffice to define a complete insertion algorithm (that is such that $x \rightarrow T \in \mathbf{S T}(n)$ if $T \in \mathbf{S T}(n)$ and $x \in \mathcal{C}_{n}$ ) for the $n$ symplectic tableaux since the first column $C^{\prime}$ of $x \rightarrow T$ may be not $n$-admissible when $x$ is greater than the greatest letter of $C_{1}$. To obtain a complete insertion algorithm we have to apply relation (9) to $C^{\prime}$. This give a column $D$ of reading $x_{1} \cdots x_{p}$. Finally we compute successively the insertions $x_{p}\left(\rightarrow x_{p-1} \cdots\left(x_{1} \rightarrow C_{2} \cdots C_{r}\right)\right)$. In the sequel we only use insertion algorithm without the contraction relation (9).

(ii) To each $w=x_{1} \cdots x_{r} \in \mathcal{C}_{n}^{*}$ of length $r$ we can associate recursively a symplectic tableau $P(w)$ by setting $P(w)=x_{1}$ if $r=1$ and $P(w)=x_{r} \rightarrow P\left(x_{1} \cdots x_{r-1}\right)$ 
otherwise. If $P(w)$ belongs to $\mathbf{S T}(m)$ with $m \geq n$, results of [15] implies that $P(w) \equiv_{m}$ $w$. Moreover $P(w)$ is the unique $m$-symplectic tableau with this property. Denote by $\sim_{m}$ the equivalence relation defined on the vertices of $G_{m}$ by $w_{1} \sim_{m} w_{2}$ if and only if $w_{1}$ and $w_{2}$ belong to the same connected component of $G_{m}$. Given two words $w_{1}$ and $w_{2}$ such that $P\left(w_{1}\right)$ and $P\left(w_{2}\right)$ belong $\mathbf{S T}(m)$ we have the equivalences

$$
w_{1} \equiv_{m} w_{2} \Leftrightarrow P\left(w_{1}\right)=P\left(w_{2}\right) \Leftrightarrow P\left(w_{1}\right) \sim_{m} P\left(w_{2}\right) .
$$

Moreover we have for any $\sigma \in W_{m}$

$$
P(\sigma(w))=\sigma(P(w))
$$

(iii) The insertion algorithm is reversible in the sense that if we know the tableau $T^{\prime}$ such that $x \rightarrow T=T^{\prime}$ and the shape of $T$ we can recover the tableau $T$ and the letter $x$. This follows from the fact that the transformations 1 to 4 are reversible. More precisely, $T^{\prime}$ has one box more than $T$. Let $y$ be the letter belonging to that box. Then if we apply transformations 1 to 4 from right to left starting from $y$, we recover $T$ and $x$.

(iv) In Section 4, we will need to find for a fixed tableau $T^{\prime}$ all the pairs $(x, T)$ where $T$ is a symplectic tableau and $x$ a letter such that $x \rightarrow T=T^{\prime}$. The outside corners of the tableau $T^{\prime}$ are the boxes $c$ of $T^{\prime}$ such that there is no box down and to the right of $c$ in $T^{\prime}$. By (iii) the pairs $(x, T)$ are obtained by applying the reverse insertion algorithm to the outside corners of $T^{\prime}$.

Example 2.3.4 Suppose $n=3$ and $T^{\prime}=$\begin{tabular}{|l|l|l}
\hline 2 & 1 & 2 \\
\hline 1 & 2 & \\
\hline 3 & & . Then by applying reverse insertion
\end{tabular}

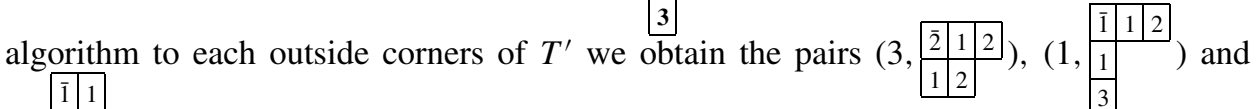
$\left(\begin{array}{lll}1, & 1 & 2\end{array}\right)$.

\section{Morris type recurrence formula}

In this section we introduce a recurrence formula for computing Kostka polynomials analogous for type $C_{n}$ to Morris recurrence formula. It allows to explain the Kostka polynomials for type $C_{n}$ as combinations of Kostka polynomials for type $C_{n-1}$. We embed type $C_{n-1}$ in type $C_{n}$ by identifying $U_{q}\left(s p_{2(n-1)}\right)$ with the sub-algebra of $U_{q}\left(s p_{2 n}\right)$ generated by the Chevalley operators $e_{i}, f_{i}$ and $t_{i}, i=0, \ldots n-2$. The weight lattice $P_{n-1}$ of $U_{q}\left(s p_{2 n}\right)$ is the $\mathbb{Z}$-lattice generated by the $\varepsilon_{i}, i=1, \ldots, n-1$ and $P_{n-1}^{+}=P_{n}^{+} \cap P_{n-1}$ is the set of dominant weights. The Weyl group $W_{n-1}$ is the sub-group of $W_{n}$ generated by the $s_{i}$, 
$i=0, \ldots n-2$ and we have $R_{n-1}^{+}=R_{n} \cap P_{n-1}$. Given any positive integer $r$, write $(r)_{n}$ for the row partition $(p, 0, \ldots 0)$ of length $n$. To obtain our recurrence formula we need to describe the decomposition $B(\gamma) \otimes B\left((r)_{n}\right)$ with $\gamma \in P_{n}^{+}$and $r>0$ an integer into its irreducible components. This is analogous for type $C_{n}$ to Pieri rule.

\subsection{Pieri rule for type $C_{n}$}

Let $\gamma=\left(\gamma_{\bar{n}}, \ldots, \gamma_{\overline{1}}\right) \in P_{n}^{+}$. By Theorem 2.2.4, the vertices of $B\left((r)_{n}\right)$ are the words

$$
L=(n)^{k_{n}} \cdots(2)^{k_{2}}(1)^{k_{1}}(\overline{1})^{k_{\overline{1}}}(\overline{2})^{k_{\overline{2}}} \cdots(\bar{n})^{k_{\bar{n}}}
$$

where $k_{i}, k_{i}$ are positive integers, $(x)^{k}$ means that the letter $x$ is repeated $k$ times in $L$ and $k_{\overline{1}}+\cdots+k_{\bar{n}}+k_{1}+\cdots+k_{n}=r$. Let $b_{\gamma}$ be the highest weight vertex of $B(\gamma)$.

Lemma 3.1.1 $b_{\gamma} \otimes L$ is a highest weight vertex of $B(\gamma) \otimes B\left((r)_{n}\right)$ if and only if the following conditions holds:

(i) $\gamma_{i}-k_{i} \geq \gamma_{\overline{i-1}}$ for $i=2, \ldots, n$ and $\gamma_{\overline{1}}-k_{1} \geq 0$,

(ii) $\gamma_{\bar{i}}-k_{i}+k_{\bar{i}} \leq \gamma_{\overline{i+1}}-k_{i+1}$ for $i=1, \ldots, n-1$.

Proof: By Lemma 2.2.1, $b_{\gamma} \otimes L$ is a highest weight vertex if and only if for any $m=$ $1, \ldots r$, each vertex $b_{\gamma} \otimes L_{m}$ (where $L_{m}$ is the word obtained by reading the $m$ leftmost letters of $L)$ is a highest weight vertex. It means that $\left(\gamma_{\bar{n}}-k_{n}, . ., \gamma_{\bar{s}}-k_{s}, \gamma_{s-1}, \ldots, \gamma_{\overline{1}}\right)$ and $\left(\gamma_{\bar{n}}-k_{n}, \ldots, \gamma_{\overline{t+1}}-k_{t+1}, \gamma_{\bar{t}}-k_{t}+k_{\bar{t}}, \ldots, \gamma_{\overline{1}}+k_{1}-k_{\overline{1}}\right)$ are partitions respectively for $s=n, \ldots, 1$ and $t=1, \ldots, n-1$. This is equivalent to the conditions

$$
\left\{\begin{array}{l}
\gamma_{\bar{s}}-k_{s} \geq \gamma_{\overline{s-1}} \text { for } s=n, \ldots, 2 \text { and } \quad \gamma_{\overline{1}}-k_{1} \geq 0 \\
\gamma_{\bar{t}}-k_{t}+k_{\bar{t}} \leq \gamma_{\overline{t+1}}-k_{t+1} \quad \text { for } t=1, \ldots, n-1
\end{array}\right.
$$

Corollary 3.1.2 $B(\gamma) \otimes B\left((r)_{n}\right)=\oplus \lambda \in P_{n}^{+} B(\lambda)^{\oplus c_{\gamma, r}^{\lambda}}$ where $c_{\gamma, r}^{\lambda}$ is the number of vertices $L \in B\left((r)_{n}\right)$ such that

(i) $k_{\bar{i}}-k_{i}=\lambda_{\bar{i}}-\gamma_{i}$ for $i=1, \ldots, n$,

(ii) $\lambda_{\bar{i}} \leq \lambda_{\overline{i+1}}-k_{\overline{i+1}}$ for $i=1, \ldots, n-1$,

(iii) $\lambda_{\bar{i}}-k_{\bar{i}} \geq \lambda \overline{i-1}+k_{i-1}-k_{\overline{i-1}}$ for $i=2, \ldots, n$ and $\lambda_{\overline{1}}-k_{\overline{1}} \geq 0$.

Proof: The multiplicity $c_{\gamma, r}^{\lambda}$ is equal to the number of highest weight vertices $b_{\gamma} \otimes L \in$ $B(\gamma) \otimes B\left((r)_{n}\right)$ of weight $\lambda$. The condition $\mathrm{wt}\left(b_{\gamma} \otimes L\right)=\lambda$ is equivalent to

$$
\gamma_{\bar{i}}-k_{i}+k_{i}^{-}=\lambda_{\bar{i}} \text { for } i=1, \ldots, n
$$


which gives (i). The assertions (ii) and (iii) are respectively obtained by replacing for any $i, \gamma_{i}$ by $\lambda_{\bar{i}}+k_{i}-k_{\bar{i}}$ in assertions (ii) and (i) of the previous lemma.

\section{Remarks}

(i) In the above corollary, $c_{\gamma, r}^{\lambda}$ is the number of ways of starting with $\gamma$, removing a horizontal strip to obtain a partition $v$ and then adding a horizontal strip to obtain $\lambda$.

(ii) $B(\gamma) \otimes B\left((r)_{n}\right)$ is not multiplicity free in general.

\subsection{Recurrence formula}

Consider $\gamma \in P_{n}^{+}$and $r$ a positive integer. We set

$$
(\gamma \otimes r)_{n}=\left\{\lambda \in P_{n}^{+}, c_{\gamma, r}^{\lambda} \neq 0\right\}
$$

with $c_{\gamma, r}^{\lambda}$ defined as in Corollary 3.1.2.

Theorem 3.2.1 Let $\mu \in P_{n}^{+}$with $n \geq 2$ and write $\mu=\left(\mu_{\bar{n}}, \mu^{\prime}\right)$ where $\mu_{\bar{n}}$ is the first part of $\mu$ and $\mu^{\prime}=\left(\mu_{\overline{n-1}}, \ldots, \mu_{\overline{1}}\right) \in P_{n-1}^{+}$. Then

$$
Q_{\mu}=\sum_{\gamma \in P_{n-1}^{+}} \sum_{r=0}^{+\infty} \sum_{m=0}^{+\infty} q^{m+r} \sum_{\lambda \in(\gamma \otimes r)_{n-1}} c_{\gamma, r}^{\lambda} K_{\lambda, \mu^{\prime}}(q) s_{\left(\mu_{\bar{n}}+r+2 m, \gamma\right)}
$$

Proof: We start from $Q_{\mu}=\left(\prod_{\alpha \in R_{n}^{+}} \frac{1}{1-q R_{\alpha}}\right) s_{\mu}$. By Proposition 3.5 of [19] we can write

$$
Q_{\mu}=\left(\prod_{\substack{\alpha \notin R_{n}^{+} \\ \alpha \notin R_{n-1}^{+}}} \frac{1}{1-q R_{\alpha}}\right)\left[\left(\prod_{R_{n-1}^{+}} \frac{1}{1-q R_{\alpha}}\right) s_{\mu}\right]
$$

Then by applying Theorem 2.1.2, we obtain

$$
Q_{\mu}=\left(\prod_{\substack{\alpha \notin R_{n}^{+} \\ \alpha \notin R_{n-1}^{+}}} \frac{1}{1-q R_{\alpha}}\right)\left(\sum_{\lambda \in P_{n-1}^{+}} K_{\lambda, \mu^{\prime}}(q) s_{\left(\mu_{\bar{n}}, \lambda\right)}\right) .
$$


Set $R_{\bar{i}}=R_{\varepsilon_{\bar{n}}-\varepsilon_{\bar{i}}}$ for $i=1, \ldots, n-1$ and $R_{i}=R_{\varepsilon_{\bar{n}}+\varepsilon_{\bar{i}}}$ for $i=1, \ldots, n$. Recall that for any $\beta \in P_{n-1}, R_{\bar{i}}(\beta)=\beta+\varepsilon_{\bar{n}}-\varepsilon_{\bar{i}}$ and $R_{i}(\beta)=\beta+\varepsilon_{\bar{n}}+\varepsilon_{\bar{i}}$. Then (14) implies

$$
\begin{aligned}
& Q_{\mu}=\sum_{\lambda \in P_{n-1}^{+}} K_{\lambda, \mu^{\prime}}(q)\left(\sum_{r=0}^{+\infty} \sum_{m=0}^{+\infty} \sum_{k_{\overline{1}}+\cdots+k_{\overline{n-1}}+k_{1}+\cdots+k_{n-1}=r} q^{m+r}\left(R_{n}\right)^{m}\left(R_{1}\right)^{k_{1}}\left(R_{\overline{1}}\right)^{k_{\overline{1}}}\right. \\
& \left.\cdots\left(R_{n-1}\right)^{k_{n-1}}\left(R_{\overline{n-1}}\right)^{k_{\overline{n-1}}} s_{\left(\mu_{\bar{n}}, \lambda\right)}\right) \text {. } \\
& Q_{\mu}=\sum_{r=0}^{+\infty} \sum_{m=0}^{+\infty} q^{m+r} \sum_{\lambda \in P_{n-1}^{+}} K_{\lambda, \mu^{\prime}}(q) \sum_{k_{\overline{1}}+\cdots+k_{\overline{n-1}}+k_{1}+\cdots+k_{n-1}=r} s_{\left(\mu_{\bar{n}}+r+2 m, \lambda_{\overline{n-1}}+k_{n-1}-k_{\overline{n-1}}\right.}, \\
& \left.\cdots, \lambda_{\overline{1}}+k_{1}-k_{\overline{1}}\right) \text {. }
\end{aligned}
$$

Fix $\lambda, m$ and $r$ and consider

$$
S=\sum_{k_{\overline{1}}+\cdots+k_{\overline{n-1}}+k_{1}+\cdots+k_{n-1}=r} S_{\left(\mu_{\bar{n}}+r+2 m, \lambda_{\overline{n-1}}+k_{n-1}-k_{\overline{n-1}}, \cdots, \lambda_{\overline{1}}+k_{1}-k_{\overline{1}}\right)}
$$

Set $\gamma=\left(\lambda_{\overline{n-1}}+k_{n-1}-k_{\overline{n-1}}, \ldots, \lambda_{\overline{1}}+k_{1}-k_{\overline{1}}\right)$.

1. Suppose first that there exists $i \in\{1, \ldots, n-2\}$ such that $\lambda_{\bar{i}}>\lambda_{\overline{i+1}}-k_{\overline{i+1}}$. Set $\tilde{\gamma}=s_{i} \circ \gamma$ that is

$$
\begin{aligned}
\tilde{\gamma}= & s_{i}\left(\gamma_{n-1}+n-1, \ldots, \gamma_{\overline{i+1}}+n-i+1, \gamma_{i}\right. \\
& \left.+n-i, \ldots, \gamma_{\overline{1}}+1\right)-(n-1, \ldots, 1) .
\end{aligned}
$$

Then $\gamma_{\bar{s}}=\tilde{\gamma}_{\bar{s}}$ for $s \neq i+1, i, \tilde{\gamma}_{\overline{i+1}}=\gamma_{\bar{i}}-1$ and $\tilde{\gamma}_{\bar{i}}=, \gamma_{i+1}+1$ that is

$$
\left\{\begin{array}{l}
\tilde{\gamma}_{\overline{i+1}}=\lambda_{\bar{i}}+k_{i}-k_{\bar{i}}-1 \\
\tilde{\gamma}_{i}=\lambda \overline{i+1}+k_{i+1}-k_{\overline{i+1}}+1
\end{array} .\right.
$$

Write $\tilde{k}_{i+1}=k_{i}, \tilde{k}_{i}=k_{i+1}, \tilde{k}_{\overline{i+1}}=\lambda_{\overline{i+1}}-\lambda_{\bar{i}}+k_{\bar{i}}+1$ and $\tilde{k}_{\bar{i}}=\lambda_{\bar{i}}-\lambda_{\overline{i+1}}+k_{\overline{i+1}}-1$. To make our notation homogeneous set $\tilde{k}_{t}=k_{t}$ for any $t \neq i, i+1, \bar{i}, \overline{i+1}$. Then $\lambda_{\bar{i}}>\lambda_{\overline{i+1}}-\tilde{k}_{\overline{i+1}}$. We have $\tilde{k}_{\overline{i+1}} \geq 0$ and $\tilde{k}_{\bar{i}}=\lambda_{\bar{i}}-\lambda \overline{i+1}+k_{\overline{i+1}}-1 \geq 0$ since $\lambda_{\bar{i}}>\lambda \overline{i+1}-k_{\overline{i+1}}$. Moreover $\tilde{k}_{\overline{1}}+\cdots+\tilde{k}_{\overline{n-1}}+\tilde{k}_{1}+\cdots+\tilde{k}_{n-1}=r$ and for any $s \in\{1, \ldots, n-2\}$

$$
\tilde{\gamma}_{\bar{s}}=\lambda_{\bar{s}}+\tilde{k}_{s}-\tilde{k}_{\bar{s}}
$$


2. Suppose that $\lambda_{\bar{i}} \leq \lambda_{\overline{i+1}}-k_{\overline{i+1}}$ for all $i=1, \ldots, n-2$ and $\lambda_{\overline{1}}-k_{\overline{1}}<0$. Set $\tilde{\gamma}=s_{0} \circ \gamma$. Then $\gamma_{\bar{s}}=\tilde{\gamma}_{\bar{s}}$ for $s \neq 1$ and $\tilde{\gamma}_{\overline{1}}=-\lambda_{\overline{1}}-k_{1}+k_{\overline{1}}-2$. Write $\tilde{k}_{i}=k_{i}, \tilde{k}_{\bar{i}}=k_{\bar{i}}$ for any $i=2, \ldots, n-1$ and set $\tilde{k}_{1}=k_{\overline{1}}-\lambda_{\overline{1}}-1, \tilde{k}_{\overline{1}}=k_{1}+\lambda_{\overline{1}}+1$. We have $\tilde{k}_{1} \geq 0, \lambda_{\bar{i}} \leq$ $\lambda_{\overline{i+1}}-\tilde{k}_{\overline{i+1}}$ for any $i=1, \ldots, n-2$ and $\lambda_{\overline{1}}-\tilde{k}_{\overline{1}}<0$. Moreover $\tilde{k}_{\overline{1}}+\cdots+\tilde{k}_{\bar{n}-1}+\tilde{k}_{1}+$ $\cdots+\tilde{k}_{n-1}=r$.

3. Now suppose that $\lambda_{\bar{s}} \leq \lambda_{\overline{s+1}}-k_{\overline{s+1}}$ for any $s \in\{1, \ldots, n-2\}, \lambda_{\overline{1}}-k_{\overline{1}} \geq 0$ and there exists $i \in\{1, \ldots, n-2\}$ such that $\lambda_{\overline{i+1}}-k_{\overline{i+1}}<\lambda_{\bar{i}}+k_{i}-k_{\bar{i}}$. Define $\tilde{\gamma}=s_{i} \circ \gamma$ as above. Set $\tilde{k}_{\overline{i+1}}=k_{\bar{i}+1}, \tilde{k}_{\bar{i}}=k_{\bar{i}}, \tilde{k}_{i+1}=\lambda_{\bar{i}}-\lambda_{\overline{i+1}}-k_{\bar{i}}+k_{i}+k_{\overline{i+1}}-1$ and $\tilde{k}_{i}=\left(\lambda_{\overline{i+1}}-\lambda_{\bar{i}}-k_{\overline{i+1}}\right)+k_{i+1}+k_{\bar{i}}+1$. Write $\tilde{k}_{t}=k_{t}$ for any $t \neq i, i+1, \bar{i}, \bar{i}+1$. We obtain $\tilde{k}_{i} \geq 0$ and $\tilde{k}_{i+1} \geq 0$ since $\lambda_{\bar{i}} \leq \lambda_{\overline{i+1}}-k_{\overline{i+1}}$ and $\lambda_{\overline{i+1}}-k_{\overline{i+1}}<\lambda_{\bar{i}}+k_{i}-k_{\bar{i}}$. Since $\tilde{k}_{\bar{s}}=k_{\bar{s}}$ for any $s=1, \ldots, n-1$, we have $\lambda_{\bar{s}} \leq \lambda_{\overline{s+1}}-\tilde{k}_{\overline{s+1}}$ for any $s \in\{1, \ldots, n-2\}$ and $\lambda_{\overline{1}}-\tilde{k}_{\overline{1}} \geq 0$. Moreover the assertion $\lambda_{\overline{i+1}}-\tilde{k}_{\bar{i}+1}<\lambda_{\bar{i}}+\tilde{k}_{i}-\tilde{k}_{\bar{i}}$ holds since it is equivalent to $0<k_{i+1}+1$. Finally $\tilde{k}_{\overline{1}}+\cdots+\tilde{k}_{\overline{n-1}}+\tilde{k}_{1}+\cdots+\tilde{k}_{n-1}=r$ and for any $s \in\{1, \ldots, n-2\}$

$$
\tilde{\gamma}_{\bar{s}}=\lambda_{\bar{s}}+\tilde{k}_{s}-\tilde{k}_{\bar{s}}
$$

Denote by $E_{1} E_{2}$ and $E_{3}$ the sets of multi-indices $\left(k_{1}, \ldots, k_{\overline{n-1}}, k_{1}, \ldots, k_{n-1}\right)$ such that $k_{\overline{1}}+\cdots+k_{\overline{n-1}}+k_{1}+\cdots+k_{n-1}=r$ and satisfying respectively the assertions $1,2,3$. Let $\chi$ be the map defined on $E_{1} \cup E_{2} \cup E_{3}$ by

$$
\chi(\gamma)=\tilde{\gamma}
$$

Then by the above arguments $\chi$ is a bijection which verifies $\chi\left(E_{i}\right)=E_{i}$ for $i=1,2,3$. Now the pairing $\gamma \leftrightarrow \tilde{\gamma}$ provides the cancellation of all the $s_{\gamma}$ with $\gamma=\left(\lambda_{\overline{n-1}}+k_{n-1}-\right.$ $\left.k_{\overline{n-1}}, \ldots, \lambda_{\overline{1}}+k_{1}-k_{\overline{1}}\right)$ such that $\left(k_{\overline{1}}, \ldots, k_{\bar{n}}, k_{1}, \ldots, k_{n}\right) \in E_{1} \cup E_{2} \cup E_{3}$ appearing in $S$. Indeed $s_{\left(\mu_{\bar{n}}+r+2 m, \gamma\right)}=-s_{\left(\mu_{\bar{n}}+r+2 m, \tilde{\gamma}\right)}$. By Corollary 3.1.2 it means that

$$
S=\sum_{\substack{\gamma \in P_{n-1}^{+} \\ \gamma \in(\gamma \oplus r)_{n-1}}} c_{\gamma, r}^{\lambda} s_{\left(\mu_{\bar{n}}+r+2 m, \gamma\right)}
$$

and the theorem is proved.

Note that the theorem is also true for $n=2$. In this case $R_{n-1}$ is the set of positive roots of the root system $A_{1}$.

Corollary 3.2.2 Let $v, \mu \in P_{n}^{+}$such that $\mu_{\bar{n}} \geq v_{\overline{n-1}}$. Suppose $v_{\bar{n}} \geq \mu_{\bar{n}}$ (otherwise $\left.K_{v, \mu}(q)=0\right)$ and set $l=v_{\bar{n}}-\mu_{\bar{n}}, v^{\prime}=\left(v_{\overline{n-1}}, \ldots, v_{\overline{1}}\right)$. Then

$$
K_{v, \mu}(q)=\sum_{r+2 m=l} q^{r+m} \sum_{\lambda \in\left(v^{\prime} \otimes r\right)_{n-1}} c_{v^{\prime}, r}^{\lambda} K_{\lambda, \mu^{\prime}}(q)
$$


Proof: Let $m, r$ be two integers such that $\left(\mu_{\bar{n}}+r+2 m, v^{\prime}\right)=v$. Then $l=r+2 m$. Consider $s_{\left(\mu_{\bar{n}}+r_{*}+2 m_{*}, \gamma\right)}$ and $s_{\left(\mu_{\bar{n}}+r+2 m, v^{\prime}\right)}$ appearing in (13). Suppose that there exists $\sigma \in W_{n}$ such that $\left(\mu_{\bar{n}}+r_{*}+2 m_{*}, \gamma\right)=\sigma \circ\left(\mu_{\bar{n}}+r+2 m, v^{\prime}\right)=\sigma\left(\mu_{\bar{n}}+r+2 m+n, v_{\overline{n-1}}+\right.$ $\left.n-1, \ldots, v_{\overline{1}}+1\right)-(n, \ldots, 1)$. We can not have $\sigma(p)=\bar{n}$ with $p \in\{1, \ldots, n\}$ otherwise $\mu_{\bar{n}}+r_{*}+2 m_{*}<0$. Set $\sigma(\bar{p})=\bar{n}$ with $p \in\{1, \ldots, n\}$. If $p<n$ we must have

$$
\nu_{\bar{p}}+p=\mu_{\bar{n}}+r_{*}+2 m_{*}+n .
$$

Thus $v_{\bar{p}}=\mu_{\bar{n}}+r_{*}+2 m_{*}+n-p>\mu_{\bar{n}}$ which contradicts the hypothesis $\mu_{\bar{n}} \geq v_{\overline{n-1}}$. Hence $\sigma(\bar{n})=\bar{n}$ and $r_{*}+2 m_{*}=l$ that is $\sigma \in W_{n-1}$. Moreover we have $\gamma=v^{\prime}$ since $w \circ v^{\prime}=\gamma$ and $\nu, \gamma \in P_{n-1}$. This proves that $s_{\left(\mu_{\bar{n}}+r+2 m, v^{\prime}\right)}$ can not be obtained by applying the straightening law for Schur functions on $s_{\left(\mu_{\bar{n}}+r_{*}+2 m_{*}, \gamma\right)}$ with $\left(\mu_{\bar{n}}+r_{*}+2 m_{*}, \gamma\right) \neq\left(\mu_{\bar{n}}+r+2 m, v^{\prime}\right)$. Then the corollary directly follows from (13) and Theorem 2.1.2.

Now suppose that $v=(p)_{n}=(p, 0, \ldots, 0) \in P_{n}^{+}$. Then by Corollary 3.2.2 for any $\mu \in P_{+}^{n}$ we must have

$$
K_{(p)_{n}, \mu}(q)=\sum_{r+2 m=l} q^{r+m} K_{(r)_{n-1}, \mu^{\prime}}(q)
$$

with $l=p-\mu_{\bar{n}}$ and $\mu^{\prime}$ defined as in statement of Theorem 3.2.1. This implies that $K_{(p)_{n}, \mu}(q)$ may be computed recursively. We are going to give an explicit formula for $K_{(p)_{n}, \mu}(q)$. The vertices of $\left.B\left((p)_{n}\right)_{\mu}=\left\{L \in B\left(p \Lambda_{n-1}\right), \operatorname{wt}(b)=\mu\right)\right\}$ are the words

$$
L=(n)^{k_{n}} \cdots(2)^{k_{2}}(1)^{k_{1}}(\overline{1})^{k_{\overline{1}}}(\overline{2})^{k_{\overline{2}}} \cdots(\bar{n})^{k_{\bar{n}}}
$$

with $\mu_{\bar{i}}=k_{\bar{i}}-k_{i}$ for $i=1, \ldots, n$ and $k_{\overline{1}}+\cdots+k_{\bar{n}}+k_{1}+\cdots+k_{n}=p$.

Proposition 3.2.3 Let $p \geq 1$ be an integer. For any $\mu \in P_{n}^{+}$we have

$$
K_{(p)_{n}, \mu}(q)=q^{f_{n}(\mu)} \sum_{L \in B\left((p)_{n}\right)_{\mu}} q^{\theta_{n}(L)}
$$

where $f_{n}(\mu)=\sum_{i=1}^{n}(n-i) \mu_{\bar{i}}$ and $\theta_{n}(L)=\sum_{i=1}^{n}(2(n-i)+1)\left(k_{\bar{i}}-\mu_{\bar{i}}\right)$.

Proof: We proceed by induction on $n$. Suppose $n=1$ we have $f_{1}(\mu)=0$. We can write $L=(1)^{p-k_{\overline{1}}}(\overline{1})^{k_{\overline{1}}}$ and $\mu_{\overline{1}}=2 k_{\overline{1}}-p$. Thus $\theta_{1}(L)=k_{\overline{1}}-\mu_{\overline{1}}=\left(p-\mu_{\overline{1}}\right) / 2$ and the proposition is true by (3).

Now suppose the proposition true for $n-1$. First note that $f_{n}(\mu)=f_{n-1}\left(\mu^{\prime}\right)+\sum_{i=1}^{n-1} \mu_{i}^{\bar{i}}$ where $\mu^{\prime}$ is defined as in statement of Theorem 3.2.1. The set of vertices obtained by erasing the letters $n$ and $\bar{n}$ in $B\left((p)_{n}\right)_{\mu}$ is the disjoint union of the $B\left((r)_{n-1}\right)_{\mu^{\prime}}$ with $r \in\{0, \ldots, l=$ $\left.p-\mu_{\bar{n}}\right)$ since the number of letters $n$ or $\bar{n}$ belonging to a vertex $L \in B\left((p)_{n}\right)_{\mu}$ is a least equal to $\mu_{\bar{n}}$. Its reflects the decomposition of $B\left((p)_{n}\right)_{\mu}$ into its $U_{q}\left(s p_{2(n-1)}\right)$-connected components. Consider $L \in B\left((p)_{n}\right)_{\mu}$ and denote by $L^{\prime}$ the vertex obtained by erasing all the letters $n$ and $\bar{n}$ in $L$. Let $r$ be such that $L^{\prime} \in B\left((r)_{n-1}\right)_{\mu^{\prime}}$. Then $r \in\{0, \ldots, l\}$ and $l-r$ 
is even since it is equal to the number of pairs $(n, \bar{n})$ erased in $L$. We set $l-r=2 m$. Then $k_{\bar{n}}=\mu_{\bar{n}}+m$.

We have

$$
\theta_{n}(L)=\theta_{n-1}\left(L^{\prime}\right)+2 \sum_{i=1}^{n-1}\left(k_{\bar{i}}-\mu_{\bar{i}}\right)+\left(k_{\bar{n}}-\mu_{\bar{n}}\right) .
$$

From the equality $r=\sum_{1 \leq i \leq n-1} \mu_{\bar{i}}+2 \sum_{1 \leq i \leq n-1}\left(k_{\bar{i}}-\mu_{\bar{i}}\right)$ we deduce

$$
\theta_{n}(L)=\theta_{n-1}\left(L^{\prime}\right)+r-\sum_{i=1}^{n-1} \mu_{\bar{i}}+m .
$$

Set

$$
K=\sum_{L \in B\left((p)_{n}\right)_{\mu}} q^{\theta_{n}(L)+f_{n}(\mu)}
$$

Then by the above arguments

$$
\begin{aligned}
K= & \sum_{r+2 m=l} \sum_{L^{\prime} \in B\left((r)_{n-1}\right)_{\mu^{\prime}}} q^{\theta_{n-1}\left(L^{\prime}\right)+r-\sum_{i=1}^{n-1} \mu_{i}+m+f_{n-1}\left(\mu^{\prime}\right)+\sum_{i=1}^{n-1} \mu_{\bar{i}}} \\
& =\sum_{r+2 m=l} q^{r+m} \times \sum_{L^{\prime} \in B\left((r)_{n-1}\right)_{\mu^{\prime}}} q^{\theta_{n-1}\left(L^{\prime}\right)+f_{n-1}\left(\mu^{\prime}\right)} .
\end{aligned}
$$

Thus we obtain by the induction hypothesis

$$
K=\sum_{r+2 m=l} q^{r+m} K_{(r)_{n-1}, \mu^{\prime}}(q)
$$

Finally $K=K_{(p)_{n}, \mu}(q)$ by (15).

Corollary 3.2.4 Write $\left(1^{2}\right)_{n}$ for the partition of length $n$ equal to $(1,1,0, \ldots, 0)$. Then

$$
K_{\left(1^{2}\right)_{n}, 0}(q)=\sum_{i=1}^{n-1} q^{2 i}
$$

To prove this corollary we need the more general lemma above

Lemma 3.2.5 Write $\left(1^{p}\right)_{n}$ for the partition of length $n(1, \ldots 1,0, \ldots, 0)$ with $p \geq 2$ parts equal to 1 . Then

$$
K_{\left(1^{p}\right)_{n}, 0}(q)=(q-1) K_{\gamma^{p}, 0}(q)+q K_{\left(1^{p}\right)_{n-1}, 0}(q)+q K_{\left(1^{p-2}\right)_{n-1}, 0}(q)
$$

where $\gamma^{p}=(2,1, \ldots, 1,0, \ldots, 0) \in P_{n-1}^{+}$contains $p-2$ parts equal to 1 . 
Proof: With $\mu=0$ formula (13) becomes

$$
Q_{0}=\sum_{\gamma \in P_{n-1}^{+}} \sum_{r=0}^{+\infty} \sum_{m=0}^{+\infty} q^{m+r} \sum_{\lambda \in(\gamma \otimes r)_{n-1}} c_{\gamma, r}^{\lambda} K_{\lambda, 0}(q) s_{(r+2 m, \gamma)}
$$

By using the straightening law for Schur functions and Theorem 2.1.2 we have to find all the $(r+2 m, \gamma)$ such that there exists $\sigma \in W_{n-1}$ satisfying $\sigma \circ(r+2 m, \gamma)=\left(1^{p}\right)_{n}$ that is

$$
\sigma\left(r+2 m+n, \gamma_{\overline{n-1}}+n-1, \ldots, \gamma_{\overline{1}}+1\right)=(n+1, \ldots, n-p+2, n-p, \ldots, 1) .
$$

We have $r+2 m+n \geq n$ hence $\sigma(\bar{n}) \in\{\bar{n}, \overline{n-1}\}$.

(i) If $\sigma(\bar{n})=\bar{n}$ then $r=1$ and $m=0$. For $k \notin\{1, n\}, \gamma_{\bar{k}}+k>1$ thus $\sigma(\overline{1})=\overline{1}$. By a straightforward induction we obtain $\sigma(\bar{k})=\bar{k}$ for $k \in\{1, \ldots, n-p\}$. Moreover we have $\gamma_{\bar{k}}+k \leq n$ for $k<n$. This implies that $\gamma_{n-1} \in\{0,1\}$ since $\gamma_{n-1}+n-1 \geq n-1$. We can not have $\gamma_{n-1}=0$ otherwise $\gamma_{\bar{k}}=0$ for any $k<n$ and the value $n$ in the left hand side of (16) is not attained. Hence $\gamma_{n-1}=1$ and $\sigma(\overline{n-1})=\overline{n-1}$. By induction we can prove that $\gamma_{n-1}=\cdots=\gamma_{\overline{n-p+1}}=1$ and $\sigma(\bar{k})=\bar{k}$ for $k \in\{n-1, \ldots, n-p+1\}$. It means that $\sigma=i d, r=1, m=0$ and $\gamma=\left(1^{p-1}\right)_{n-1}$.

(ii) If $\sigma(\bar{n})=\overline{n-1}$ then $R=m=0$. By using similar arguments than above we obtain $\gamma_{n-1}=2, \gamma_{n-2}=\cdots=\gamma_{n-p+1}=1$ and $\gamma_{\overline{n-p}}=\cdots \gamma_{\overline{1}}=0$. It means that $\sigma=s_{n}$ and $\gamma=\gamma^{p}$. Note that $s_{\left(0, \gamma^{p}\right)}=-s_{\left(\left(1^{p}\right)_{n}\right.}$ since $s_{n} \circ\left(0, \gamma^{p}\right)=\left(1^{p}\right)_{n}$ and $l\left(s_{n}\right)=1$.

Finally by Theorem 2.1.2 we must have

$$
\begin{aligned}
K_{\left(1^{p}\right)_{n}, 0}(q) & =q \times \sum_{\lambda \in\left(\left(1^{p-1}\right)_{n-1} \otimes 1\right)_{n-1}} c_{\left(1^{p-1}\right)_{n-1}, 1}^{\lambda} K_{\lambda, 0}(q)-K_{\gamma^{p}, 0}(q) \\
& =(q-1) K_{\gamma^{p}, 0}(q)+q K_{\left(1^{p}\right)_{n-1}, 0}+q K_{\left(1^{p-2}\right)_{n-1}, 0} .
\end{aligned}
$$

Proof: (of Corollary 3.2.4). We proceed by induction on $n$. For $n=2, K_{\left(1^{2}\right), 0}(q)=q^{2}$. Suppose the corollary true for $k<n$. Then by applying Lemma 3.2.5 we obtain

$$
K_{\left(1^{2}\right)_{n}, 0}(q)=(q-1) K_{(2)_{n-1}, 0}(q)+q K_{\left(1^{2}\right)_{n-1}, 0}(q)+q .
$$

It follows from Proposition 3.2.3 that

$$
K_{(2)_{n-1}, 0}(q)=\sum_{i=1}^{n-1} q^{2 i-1}
$$


Thus

$$
\begin{aligned}
K_{\left(1^{2}\right)_{n}, 0}(q)= & (q-1) \sum_{i=1}^{n-1} q^{2 i-1}+q \sum_{i=1}^{n-2} q^{2 i}+q=\sum_{i=1}^{n-1} q^{2 i}-\sum_{i=1}^{n-1} q^{2 i-1} \\
& +\sum_{i=1}^{n-2} q^{2 i+1}+q=\sum_{i=1}^{n-1} q^{2 i}
\end{aligned}
$$

Note that we can not deduce an explicit formula for $K_{\left(1^{p}\right)_{n}, 0}(q)$ with $p>2$ from the recurrence formula of Lemma 3.2.5 as we have done in Proposition 3.2.3 since we have no explicit formula for $K_{\gamma^{p}, 0}(q)$ as soon as $p>2$. Nevertheless we will give a conjectural general formula for $K_{\left(1^{p}\right)_{n}, 0}(q)$ in Section 5 .

\section{Cyclage graphs for symplectic tableaux}

Given a symplectic tableau $T \in \mathbf{S T}(n)$, we can factorize $w(T)$ in a unique way by setting $w(T)=x u$ where $u$ is a word and $x$ is a letter. It is easy to verify that $u$ is also the reading of a symplectic tableau, say $T_{*} \in \mathbf{S T}(n)$. The initial cocyclage operation on $T$ consists of the insertion $x \rightarrow T_{*}$. We are going to see that all the initial cocyclage operations are not relevant for defining a charge.

It follows from Paragraph 2.3 that the tableau obtained by cocycling a tableau $T \in \mathbf{S T}(n)$ does not belong to $\mathbf{S T}(n)$ in general but belongs to $\mathbf{S T}(n+1)$. To overcome this problem we are going to define our cocyclage operation directly on the complete symplectic tableaux set $\mathbf{S T}=\cup_{n \geq 1} \mathbf{S T}(n)$.

\subsection{Cocyclage operation}

Set $\mathcal{C}_{\infty}=\cup_{n \geq 1} \mathcal{C}_{n}$. Then $\mathcal{C}_{\infty}$ is totally ordered by $\leq$. Given any $T \in \mathbf{S T}$ there exists an integer $m \geq 1$ such that $T \in \mathbf{S T}(m)$. Recall that $d_{\bar{i}}$ is the number of letters $\bar{i}$ of $T$ minus the number of letters $i$. For any weight $\mu \in P_{n}$, we will say that $T \in \mathbf{S T}$ is a tableau of weight $\mu$ if $T \in \mathbf{S T}(m)$ with $m \geq n, d_{\bar{i}}=0$ for $i>n$ and $d_{\bar{k}}=\mu_{\bar{k}}$ for $k=1, \ldots, n$. For any $\mu \in P_{n}$, the set of tableaux of weight $\mu$ is denoted $\mathbf{S T}(\mu)$. If $T \in \mathbf{S T}(\mu)$, the number of letters $k$ with $k>n$ which belong to $T$ is equal to the number of letters $\bar{k}$.

Let $w \in \mathcal{C}_{\infty}^{*}$ and write $w=x u$ with $x$ a letter and $u \in \mathcal{C}_{\infty}^{*}$. The cocyclage shift $\xi$ is the map defined on $\mathcal{C}_{\infty}^{*}$ by

$$
\xi(w)=u x
$$

Lemma 4.1.1 For any $n \geq 1, \sigma \in W_{n}$, and $w \in \mathcal{C}_{\infty}^{*}, \xi(\sigma(w))=\sigma(\xi(w))$.

Proof: The proof is analogous to that of Proposition 5.6.1 of [16]. 
Consider a symplectic tableau $T=C_{1} \cdots C_{r} \in \mathbf{S T}(m)$ with $r>1$. We will say that the cocyclage operation is authorized for $T$ if there is no letter $y \in \mathcal{C}_{m}$ such that $y \in C_{i}$ for all $i=1, \ldots, r$ and $\bar{y} \notin T$. Thus, since each column of $T$ contains at most one letter $\bar{y}$, the cocyclage operation is not authorized for $T$ when there exists an integer $p \in\{1, \ldots, m\}$ such that $\left|d_{\bar{p}}\right|$ is equal to $r$. If the cocyclage operation is authorized for $T$, we write $w(T)=x w\left(T_{*}\right)$ where $T_{*} \in \mathbf{S T}(m)$ and $x \in \mathcal{C}_{m}$. and we set

$$
U(T)=x \rightarrow T_{*} .
$$

\section{Remarks}

(i) $U(T)$ belongs to $\mathbf{S T}$ and $\operatorname{wt}(U(T))=\operatorname{wt}(T)$. More precisely, if $T \in \mathbf{S T}(m)$ then $U(T) \in \mathbf{S T}(m)$ if the heights of the first columns of $T$ and $U(T)$ are equal, and otherwise $U(T) \in \mathbf{S T}(m+1)$.

(ii) If $\operatorname{wt}(T)=0$ then the cocyclage operation is always authorized.

(iii) By convention there is no cocyclage operation on the columns.

(iv) Suppose that $T$ contains $r$ columns. Then the number of columns of $T_{*}$ is $r$ or $r-1$. Thus $U(T)$ contains at most $r+1$ columns since its shape is obtained by adding one box to that of $T_{*}$. Moreover $U(T)$ contains $r+1$ columns only if the height of its rightmost column is equal to 1 .

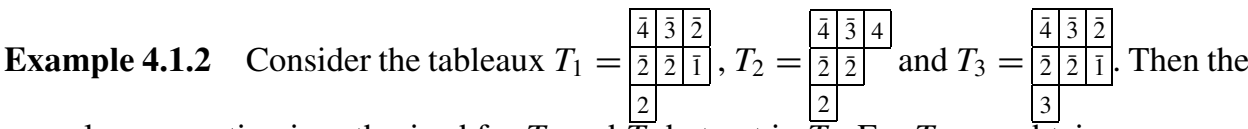
cocyclage operation is authorized for $T_{1}$ and $T_{2}$ but not in $T_{3}$. For $T_{1}$, we obtain

$$
x_{1, *}=\overline{2}, T_{1, *}=\begin{array}{|l|l|l|}
\hline \overline{4} & \overline{3} & \overline{1} \\
\hline \overline{2} & \overline{2} & \\
\cline { 1 - 1 } 2 &
\end{array}
$$

and

$$
U\left(T_{1}\right)=\begin{array}{|l|l|l|}
\hline \overline{4} & \overline{3} & \overline{1} \\
\hline \overline{3} & \overline{2} & \\
\hline \overline{2} & 3 & \\
\hline
\end{array}
$$

Similarly we have

$$
x_{2, *}=4, T_{2, *}=\begin{array}{|l|l|}
\hline \overline{4} & \overline{3} \\
\hline \overline{2} & \overline{2} \\
\hline 2 & \\
\hline
\end{array}
$$


and

$$
U\left(T_{2}\right)=\begin{array}{l|l|l|}
\hline \overline{4} & \overline{3} \\
\hline \overline{2} & \overline{2} \\
\hline 2 & \\
\hline 4 &
\end{array} .
$$

Lemma 4.1.3 Suppose $T \in \mathbf{S T}(m)$ and consider $\sigma \in W_{m}$. Then the cocyclage operation is authorized for $T$ if and only if it is authorized for $\sigma(T)$. In this case we have $U(\sigma(T))=$ $\sigma(U(T))$.

Proof: The first statement follows from $\operatorname{wt}(T)=\operatorname{wt}(U(T))$ and the second statement follows from Lemma 4.1.1 and (12).

\subsection{Cyclage graphs}

We endow the set $\mathbf{S T}$ with a structure of graph by drawing an array $T \rightarrow T^{\prime}$ if and only if the cocyclage operation is authorized on $T$ and $U(T)=T^{\prime}$. Write $\Gamma(T)$ for the connected component containing $T$. Let $t$ be the translation operation on letters of $\mathcal{C}_{\infty}$ defined by $t(k)=k+1$ and $t(\bar{k})=\overline{k+1}$ for $k \geq 1$. We write $t(w)$ (resp. $t(T))$ for the word (resp. the tableau) obtained by applying $t$ to each letter of $w \in \mathcal{C}_{\infty}^{*}$ (resp. to each letter of $T \in$ ST).

\section{Lemma 4.2.1}

(i) Suppose $T \in \mathbf{S T}(m)$. Then $\Gamma(T)$ and $\Gamma(\sigma(T))$ are isomorphic for any $\sigma \in W_{m}$.

(ii) The cyclage graphs $\Gamma(T)$ and $\Gamma(t(T))$ are isomorphic.

(iii) Suppose that $T_{1} \neq T_{2} \in \Gamma(T)$ are such that $U\left(T_{1}\right)=U\left(T_{2}\right)=T$. Then $T_{1}$ and $T_{2}$ have different shapes.

Proof: Assertion (i) follows immediately from Lemma 4.1.3.

Let $w_{1}$ and $w_{2}$ be two words of $\mathcal{C}_{m}$. Then $w_{1} \equiv_{m} w_{2}$ if and only if $t\left(w_{1}\right) \equiv_{m+1} t\left(w_{2}\right)$. This implies that $P(t(w))=t(P(w))$ for any word $w \in \mathcal{C}_{\infty}$. Hence $t$ commutes with $U$. Since $t$ is a bijection, it is also an isomorphism between $\Gamma(T)$ and $\Gamma(t(T))$ which proves (ii).

Suppose that $T_{1}, T_{2} \in \mathbf{S T}(m)$ have the same shape $X$. Write $w\left(T_{1}\right)=x w(R)$ and $w\left(T_{2}\right)=$ $y w(S)$ with $x, y$ two letters and $R, S$ two symplectic tableaux. Then $w(R) x \equiv_{m+1} w(S) y$ since $P(w(R) x)=P(w(S) y)=T$. Then $R$ and $S$ have the same shape $Y$ obtained by deleting one box in the rightmost column of $X$. The highest weight vertices of the connected components of $G_{m+1}$ containing $w(R) x$ and $w(S) y$ may be respectively written $w\left(Y_{0}\right) x_{0}$ and $w\left(Y_{0}\right) y_{0}$ where $Y_{0} \in \mathbf{S T}(m+1)$ is the highest weight tableau of shape $Y$. The congruence $w(R) x \equiv_{m+1} w(S) y$ implies the congruence $w\left(Y_{0}\right) x_{0} \equiv_{m+1} w\left(Y_{0}\right) y_{0}$. Thus we must have $\operatorname{wt}\left(w\left(Y_{0}\right) x_{0}\right)=\operatorname{wt}\left(w\left(Y_{0}\right) x_{0}\right)$. It means that $x_{0}=y_{0}$. Hence $w(R) x$ and $w(S) y$ are congruent 
and belong to the same connected component. This implies that $w(R) x=w(S) y$, thus $x w(R)=y w(S)$ and $T_{1}=T_{2}$. So (iii) is proved.

Assertion (i) of the above lemma permits to restrict to the cyclage graphs $\Gamma(T)$ with $T \in \mathbf{S T}(\mu)$ and $\mu \in P_{n}^{+}$. Suppose first that $\mu=0$. By remark (ii) above, the cocyclage operation is always authorized on symplectic tableaux of weight 0 with at least two columns. So we can define from $T$ a sequence $\left(T_{n}\right)$ of symplectic tableaux by setting $T_{0}=T$ and $T_{k+1}=U\left(T_{k}\right)$ while $T$ is not a column.

Proposition 4.2.2 Let $T_{0} \in \mathbf{S T}(0)$ and let $T_{k+1}=U\left(T_{k}\right)$. Then the sequence $\left(T_{n}\right)$ is finite without repetition and there exists an integer $e$ such that $T_{e}$ is a column of weight 0 .

To prove this proposition we need two technical lemmas. Given two words $w_{1}, w_{2} \in \mathcal{C}_{\infty}^{*}$, write $w_{1} \triangleleft w_{2}$ if $w_{1}$ and $w_{2}$ can respectively be written $w_{1}=u_{1} x_{1} v$ and $w_{2}=u_{2} x_{2} v$ where $u_{1}, u_{2}, v \in \mathcal{C}_{\infty}^{*}$ and $x_{1}, x_{2} \in \mathcal{C}_{\infty}$ satisfy $x_{1}<x_{2}$. It means that $\triangleleft$ is the inverse lexicographic order on words of $\mathcal{C}_{\infty}^{*}$. For any symplectic tableau $T$ with $r>1$ columns, we denote by $N_{r}(T)$ the number of boxes belonging to the $r-1$ rightmost columns of $T$.

Lemma 4.2.3 Consider $\mu \in P_{n}^{+}$and $\tau \in \mathbf{S T}(\mu)$ a tableau with $r>1$ columns. Let $T, T^{\prime}$ two tableaux of $\Gamma(\tau)$ such that $T=U^{(i)}(\tau)$ and $T^{\prime}=U^{(i+1)}(\tau)$ with $i \geq 0$ an integer. Then the following assertions hold.

1. For any $j, S=U^{(j)}(\tau)$ contains at most $r+1$ columns and if it contains $r+1$ columns, the height of its rightmost column is equal to 1.

2. Suppose that $T$ contains $r$ or $r+1$ columns and $N_{r}(T)=N_{r}\left(T^{\prime}\right)$. Then only one of the following situations can happen:

(i) $T$ and $T^{\prime}$ contain $r$ columns and their $r$-th columns have the same height.

(ii) $T$ contains $r$ columns and $T^{\prime}$ contains $r+1$ columns.

(iii) $T$ and $T^{\prime}$ contains $r+1$ columns.

(iv) $T$ contains $r+1$ columns, $T^{\prime}$ contains $r$ columns and the height of the last column $C_{r}^{\prime}$ of $T^{\prime}$ is equal to $h\left(C_{r}\right)+1$.

Moreover in each case we can write $w(T)=x_{*} w\left(T_{*}\right)$ and $w\left(T^{\prime}\right)=x_{*}^{\prime} w\left(T_{*}^{\prime}\right)$ with $w\left(T_{*}^{\prime}\right) \triangleleft w\left(T_{*}\right)$.

3. Suppose that $T$ contains $r$ or $r+1$ columns and $N_{r}(T) \neq N_{r}\left(T^{\prime}\right)$. Then $N_{r}(T)>N_{r}\left(T^{\prime}\right)$.

\section{Proof:}

1. We proceed by induction on $j$ starting from $j=0$. If $S=U^{(j)}(\tau)$ contains at most $r$ columns, then by Remark (iv) before Example 4.1.2 the assertion is true for $U(S)$. Now if $S=U^{(j)}(\tau)$ contains $r+1$ columns, then $S_{*}$ contains $r$ columns and $U(S)$ contains either $r$ columns either $r+1$ columns with only one box in its rightmost column. 
2. In case (i) the box which is added to $T_{*}$ during the insertion $x_{*} \rightarrow T_{*}$ appears on the bottom of the last column $C_{r, *}$ (eventually empty) of $T_{*}$. This insertion can be written $x_{*} \rightarrow T_{*}=\left(x_{*} \rightarrow C_{1} \cdots C_{r-1}\right) C_{r, *}=\left(C_{1}^{\prime} \cdots C_{r-1}^{\prime}\right)\left(y \rightarrow C_{r, *}\right)$ that is, $x_{*}$ is first inserted in the sub-tableau composed of the $r-1$ leftmost columns of $T_{*}$ which gives a new tableau $C_{1}^{\prime} \cdots C_{r-1}^{\prime}$ and a letter $y$. This letter is then inserted on the bottom of $C_{r, *}$. Suppose that there exists an integer $i \in\{1, \ldots, r-1\}$ such that $C_{i}^{\prime} \neq C_{i}$. Then if we choose $i$ minimal we have $w\left(C_{i}^{\prime}\right) \triangleleft w\left(C_{i}\right)$ by (10) and finally $w\left(T_{*}^{\prime}\right) \triangleleft w\left(T_{*}\right)$. Now if $C_{i}^{\prime}=C_{i}$ for $i=1, \ldots, r-1$ we have $y=x_{*}$ and $x_{*} \in C_{i}, \bar{x}_{*} \notin C_{i}$ for any $i=1, \ldots, r-1$. So the letter $x_{*}$ belongs to all the columns of $T$. Then $x_{*}$ is a barred letter since $\mu \in P_{n}^{+}$and $r>1$. Moreover $\bar{x}_{*} \notin C_{r}^{*}$ for $x_{*} \rightarrow C_{r}^{*}$ is a column. Thus $\bar{x}_{*} \notin T$. This contradicts the fact that the cocyclage operation is authorized for $T$.

In case (ii) a new column of height 1 is added to the shape of $T_{*}$. The insertion can be written $x_{*} \rightarrow T_{*}=\left(x_{*} \rightarrow C_{1} \cdots C_{r-1}\right) C_{r, *}=\left(C_{1}^{\prime} \cdots C_{r-1}^{\prime}\right)\left(y \rightarrow C_{r, *}\right)=$ $C_{1}^{\prime} \cdots C_{r-1}^{\prime} C_{r, *}^{\prime} x_{*}^{\prime}$ that is, $x_{*}$ is inserted in the sub-tableau composed of the $r-1$ leftmost columns of $T_{*}$ which gives a new tableau $C_{1}^{\prime} \cdots C_{r-1}^{\prime}$ and a letter $y$. This letter is then inserted in $C_{r, *}$ which gives the column $C_{r, *}^{\prime}$ and the letter $x_{*}^{\prime}$. If $y \neq x_{*}$, we terminate as in case (i). Otherwise we have $C_{i}^{\prime}=C_{i}$ for any $i=1, \ldots, r-1$. We can not have $x_{*}^{\prime}=x_{*}$ since it would imply that $x_{*} \in C_{r, *}$ which is impossible since $C_{r}$ can not contain two letters $x_{*}$. Thus $x_{*}^{\prime}>x_{*}, w\left(C_{r, *}^{\prime}\right) \triangleleft w\left(C_{r, *}\right)$ and finally $w\left(T_{*}^{\prime}\right) \triangleleft w$ $\left(T_{*}\right)$.

Case (iii) is similar to case (i) with $h\left(C_{r}\right)=1$.

In case (iv) $C_{r+1}$ contains only the letter $x_{*}$ and a new box appears on the bottom of the column $C_{r}$ of $T_{*}$ during the insertion $x_{*} \rightarrow T_{*}$. The insertion can be written $x_{*} \rightarrow T_{*}=x_{*} \rightarrow\left(C_{1} \cdots C_{r}\right)=\left(C_{1}^{\prime} \cdots C_{r-1}^{\prime}\right)\left(y \rightarrow C_{r, *}\right)=C_{1}^{\prime} \cdots C_{r-1}^{\prime} C_{r, *}^{\prime}$, that is $x_{*}$ is inserted in the sub-tableau composed of the $r-1$ leftmost columns of $T_{*}$ which gives the tableau $\left(C_{1}^{\prime} \cdots C_{r-1}^{\prime}\right)$ and the letter $y$. This letter is then inserted on the bottom of $C_{r, *}$ which gives the column $C_{r, *}^{\prime}$. Suppose that $y=x_{*}$. We must have $x_{*} \in C_{i}$, and $\bar{x}_{*} \notin C_{i}$ for any $i=1, \ldots, r-1$. Then $x_{*}=\bar{q}$ with $q \geq 1$ that is, is a barred letter as in (i). Moreover $\bar{x}_{*}, x_{*} \notin C_{r}$ because $x_{*} \rightarrow C_{r}$ is a column. Thus $d_{\bar{q}}(w(T))=r$. Now $T$ contains $r+1$ columns, hence $T \neq \tau$. Let $j$ minimal such that $R=U^{(i-j)}(\tau)$ contains $r$ columns. Then $d_{\bar{q}}(w(R))=d_{\bar{q}}(w(T))=r$. Thus the cocyclage operation in not authorized in $R$ and we obtain a contradiction. It means that $y \neq x_{*}$. So we can terminate as in case (i).

3. It is clear from the definition of the cocyclage operation.

Lemma 4.2.4 Let $T=C_{1} \cdots C_{p} \in \mathbf{S T}(0)$ with $r>1$ columns. Then there exists an integer $k$ such that $T_{k}$ has at most $r-1$ columns. Moreover if $k$ is minimal the sequence $T_{0}, \ldots, T_{k}$ is without repetition.

Proof: Let $m \geq 1$ be an integer such that $T_{0}, \ldots, T_{m}$ have $r$ or $r+1$ columns and $N_{r}\left(T_{i}\right)=N_{r}(T)$ for any $i=1, \ldots, m$. Then by Lemma 4.2 .3 we can write $w\left(T_{i}\right)=$ 
$x_{i, *} w\left(T_{i, *}\right), i=0, \ldots, m$ with

$$
w\left(T_{m, *}\right) \triangleleft \cdots \triangleleft w\left(T_{1, *}\right) \triangleleft w\left(T_{0, *}\right) .
$$

This implies that the sequence $T_{0}, \ldots, T_{m}$ is without repetition. Now suppose that $T \in$ ST $(n)$. Then, by Remark (i) after Lemma 4.1.1 and the fact that $N_{r}\left(T_{i}\right)=N_{r}(T)$ the height of the first column of any tableau $T_{i}, i=0, \ldots, m$ is always equal to that of $T_{0}$. Thus $T_{i} \in \mathbf{S T}(n)$ for any $i=0, \ldots, m$. Denote by $p$ the number of boxes in $T_{0}$. Since the number of symplectic tableaux with $p$ boxes belonging to $\mathbf{S T}(n)$ is finite there exists an integer $s_{1}$ minimal such that $N_{r}\left(T_{s_{1}}\right)=N_{r}(T)+1$ or $T_{s_{1}}$ has at most $r-1$ columns. Then the sequence $T_{0}, \ldots, T_{s_{1}}$ is without repetition. If $T_{s_{1}}$ has at most $r-1$ columns we take $k=s_{1}$. Otherwise $T_{s_{1}}$ has $r$ or $r+1$ columns and we can obtain similarly starting from $T_{s_{1}}$ an integer $s_{2}$ minimal such that $N_{p}\left(T_{s_{2}}\right)=N_{p}\left(T_{s_{1}}\right)+1$ or $T_{s_{2}}$ has at most $r-1$ columns. The sequence $T_{s_{1}}, \ldots, T_{S_{2}}$ is without repetition. Then the sequence $T_{0}, \ldots, T_{s_{2}}$ is also without repetition. Indeed a tableau $T_{i}$ with $i \in\left\{0, \ldots, s_{1}-1\right\}$ can not be equal to a tableau $T_{j}$ with $j \in\left\{s_{1}, \ldots, s_{2}-1\right\}$ since $N_{r}\left(T_{i}\right) \neq N_{r}\left(T_{j}\right)$. By induction we can construct $T_{s_{j+1}}$ from $T_{s_{j}}$ while $T_{s_{j}}$ has $r$ or $r+1$ columns, such that $N_{r}\left(T_{s_{j+1}}\right)=N_{r}\left(T_{s_{j}}\right)+1$ and the sequence $T_{0}, \ldots, T_{s_{j+1}}$ is without repetition. The procedure terminates since the number of boxes belonging to the columns $r$ and $r+1$ decreases by 1 to each step. So the lemma is proved.

Proof: (of Proposition 4.2.2): Let $r>1$ be the number of columns of $T$. By Lemma 4.2.4, we can obtain from $T=T_{0}$ a tableau $T_{k_{1}}$ with at most $r-1$ columns and such that the sequence $T_{0}, \ldots, T_{k_{1}}$ is without repetition. If $r-1>1$ we can obtain a tableau $T_{k_{2}}$ from $T_{k_{1}}$ with at most $r-2$ columns and such that the sequence $T_{0}, \ldots, T_{k_{2}}$ is without repetition. We can define $T_{k_{s+1}}$ from $T_{k_{s}}$ while $r-s>1$ such that the sequence $T_{0}, \ldots, T_{k_{s+1}}$ is without repetition. It is clear that the procedure terminates when $T_{k_{s}}=T_{e}$ is a column of weight 0 .

It follows from Proposition 4.2.2 that $\left.\operatorname{wt}\left(T_{1}\right)=\operatorname{wt}\left(T_{2}\right) \nRightarrow T_{1}\right)=\Gamma\left(T_{2}\right)$ in general. For example all the columns of weight 0 occur in different connected components.

We give below

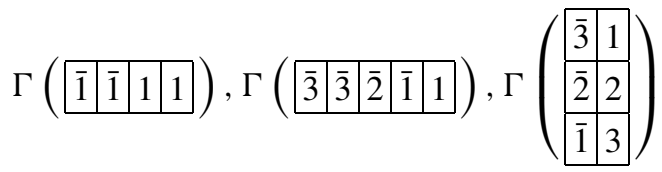

and

$$
\Gamma\left(\begin{array}{l|l}
\hline \overline{2} & 1 \\
\hline \overline{1} & 2 \\
\hline
\end{array}\right)
$$




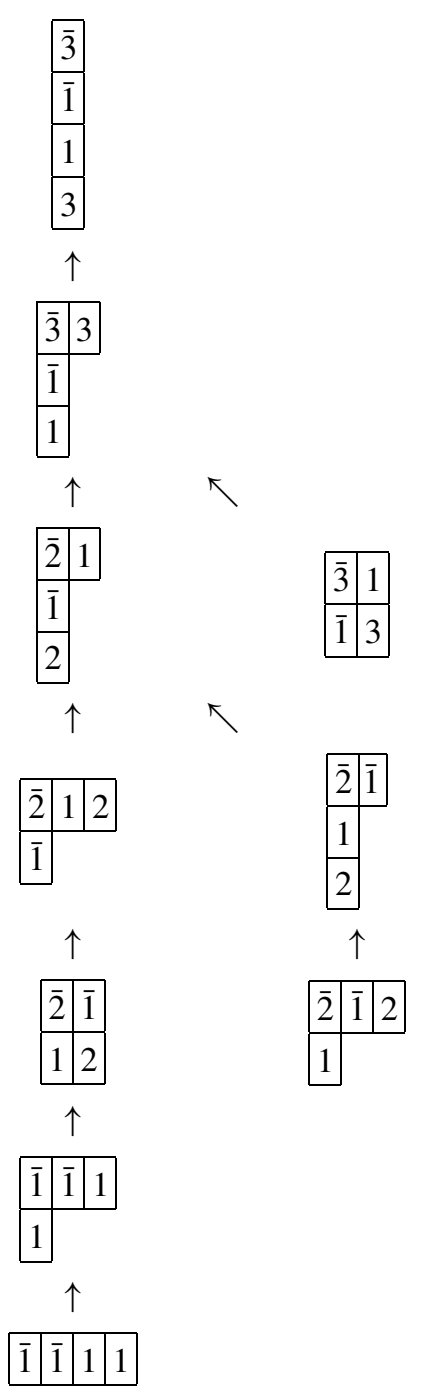




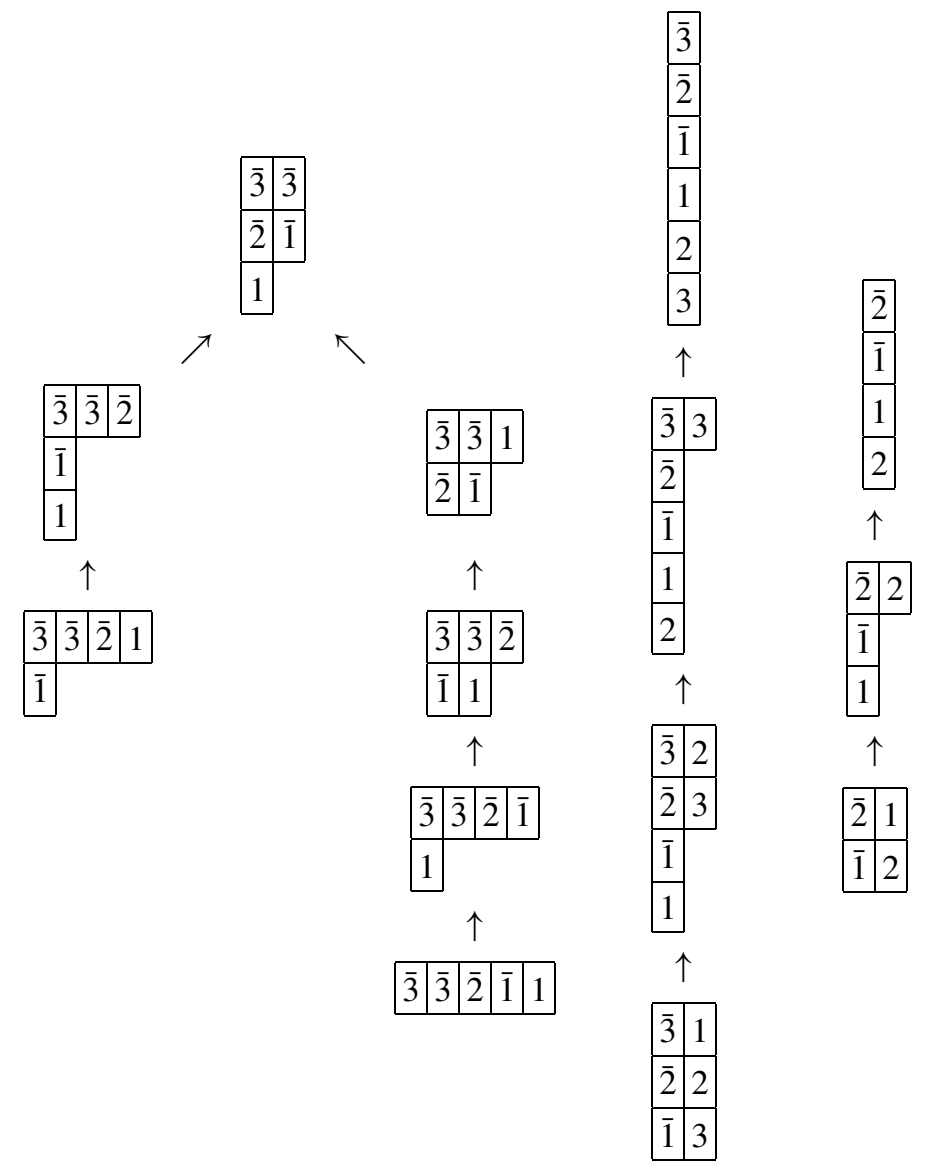

\section{Remarks}

(i) Given $T^{\prime} \in \mathbf{S T}$, it is possible to find the tableaux $T$ (if there is any) such that $U(T)=$ $T^{\prime}$. To do this we find all the pairs $\left(x, T_{*}\right)$ obtained by applying the reverse insertion algorithm on the outside corners of $T^{\prime}$. By definition of $U$, the tableaux $T$ are precisely those which verify $w(T)=x w\left(T_{*}\right)$ for a pair $\left(x, T_{*}\right)$. They are determined by the pairs $\left(x, T_{*}\right)$ for which $x w\left(T_{*}\right)$ is the reading of a symplectic tableau. For example $T^{\prime}=$\begin{tabular}{|l|l|}
\hline 2 & 1 \\
\hline $\begin{array}{l}1 \\
1\end{array}$ & 2 \\
\hline
\end{tabular} has only one outside corner which gives $x=\overline{1}$ and $T_{*}=$\begin{tabular}{l|l|l|l|}
$\overline{1}$ & 1
\end{tabular} . There is no tableau $T$ such that $U(T)=T^{\prime}$ since $\overline{1}(1 \overline{1} 1)$ is not the reading of a symplectic tableau.

(ii) In the definition of $U(T)$ we have restricted to the authorized cocyclages. For type $A$ the cyclage graphs take also into account non initial cyclages. When $T$ is of dominant evaluation that is $\operatorname{wt}(T)$ is a dominant weight, they are obtained by considering all 
the factorizations $w(T) \equiv y w(Y)$ in the plactic monoid with $y \neq 1$ a letter and $Y$ a semi-standard tableau. The use of non initial cocyclages with symplectic tableaux is problematic because it can make appear loops in the cyclage graphs. For example consider $Z=$\begin{tabular}{ll}
$\overline{3}$ & 3 \\
\hline$\overline{1}$ & \\
\hline 1
\end{tabular} . Then $w(Z)=(3 \overline{3} \overline{1}) 1 \equiv_{3} \overline{2}(2 \overline{1} 1) \equiv_{3} \overline{2} \overline{1} 21$. Now if we compute

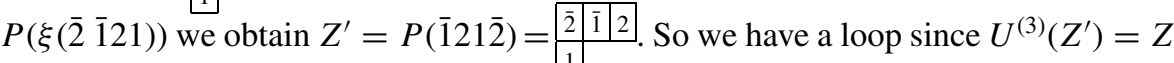
(see the cyclage graph $\Gamma\left(\begin{array}{llll}\overline{1} & \overline{1} & 1 & 1\end{array}\right)$ above).

(iii) For type $A$, every semi-standard tableau belongs to the cyclage graph containing a row tableau. By considering $\Gamma\left(\begin{array}{c|c}\frac{1}{2} & 1 \\ \hline & 2\end{array}\right)$ we see that such a property is false with the symplectic tableaux even if we consider non initial cyclages. This explains why we have to consider the cocyclage operation and not the cyclage one.

\subsection{Reduction operations}

Consider $T \in \mathbf{S T}(\mu)$ with $\mu \in P_{n}^{+}$. If the cocyclage operation is not authorized for $T$, then $T$ does not contain any letters $n$. Indeed there exists $p \in\{1, \ldots n\}$ such that $\mu_{\bar{p}}$ is equal to the number of columns of $T$. Thus $\mu_{\bar{n}}=\mu_{\bar{p}}$ since $\mu_{\bar{n}} \geq \mu_{\bar{p}}$. So each column of $T$ contains a letter $\bar{n}$ and no letter $n$. Let $T_{\$}$ be the tableau obtained first by erasing the letters $\bar{n}$ in $T$ next by applying $t$ to the letters $x \in T$ such that $\bar{n}<x<n$. It is easy to verify that $T_{\$} \in \mathbf{S T}\left(\mu^{\prime}\right)$ with $\mu^{\prime}=\left(\mu_{\overline{n-1}}, \ldots, \mu_{\overline{1}}, 0\right) \in P_{n}^{+}$. Now if the cocyclage operation is not authorized on $T_{\$}$, we can compute $\left(T_{\$}\right)_{\$}$ and so on to obtain a symplectic tableau $\hat{T}$ which is either a column of weight 0 (eventually empty) either a symplectic tableau for which the cocyclage operation is authorized. We will say that $\hat{T}$ is obtained by reduction operations from $T$. By convention we set $\hat{T}=T$ if the cocyclage operation is already defined for the symplectic tableau $T$.

Remark When a reduction operation is done in $T \in \mathbf{S T}(\mu)$ with $\mu \in P_{n}^{+}, \mu_{\bar{n}}$ is equal to the first part of the shape of $T$. To define a charge statistic on symplectic tableaux related to Kostka-Foulkes polynomials, it seems natural by Lemma 2.1.3 to impose that the charges associated to $T$ and $\hat{T}$ should be equal as we will do in Section 5 .

From $T \in \mathbf{S T}(\mu)$ we can compute a sequence of symplectic tableaux by setting $T_{0}=T$ and

$$
T_{k+1}=\left\{\begin{array}{ll}
U\left(\hat{T}_{k}\right) & \text { if } U\left(\hat{T}_{k}\right) \text { is not a column } \\
\left(\widehat{\left.U\left(\hat{T}_{k}\right)\right)}\right. & \text { otherwise }
\end{array} .\right.
$$

while $\hat{T}_{k}$ is not a column.

Proposition 4.3.1 The sequence $\left(T_{n}\right)$ is finite without repetition and the last symplectic tableau obtained is a column of weight 0 (eventually empty).

Proof: Suppose first that there is a loop in the sequence $\left(T_{n}\right)$ that is, there exists two integers $k$ and $s$ such that $T_{k}=T_{k+s}$. Then $T_{i}=\hat{T}_{i}$ for any $i=k, \ldots, k+s-1$. Choose 
$p \in\{k, . ., k+s-1\}$ such that the number of columns of $T_{p}$ is minimal among all the tableaux $T_{i}, i=k, \ldots, k+s-1$. Denote by $r$ the number of columns of $T_{p}$. Then by assertion 1 of Lemma 4.2.3, $T_{k}, \ldots, T_{k+s-1}$ contain $r$ or $r+1$ columns since every $T_{i}$, $i=k, \ldots, k+s-1$ can be obtained by cocyclage operations from $T_{p}$. We must have $N_{r}\left(T_{k}\right) \leq \cdots \leq N_{r}\left(T_{k+s}\right)$. This implies that $N_{r}\left(T_{k}\right)=\cdots=N_{r}\left(T_{k+s-1}\right)$ for $T_{k+s}=T_{k}$. Then by assertion 2 of Lemma 4.2.3 we can write $w\left(T_{i}\right)=x_{i, *} w\left(T_{i, *}\right), i=k, \ldots, k+s$ with

$$
w\left(T_{k+s, *}\right) \triangleleft \cdots \triangleleft w\left(T_{s, *}\right) .
$$

We obtain a contradiction since $w\left(T_{k+s, *}\right)=w\left(T_{s, *}\right)$. It means that there is no loop in the sequence $\left(T_{n}\right)$. Hence this sequence is without repetition.

Now suppose that this sequence is infinite. Then there exists an integer $a$ such that the sequence $\left(T_{n+a}\right)_{n \geq 0}$ is infinite without reduction operation. In the proof of Lemma 4.2.4 the hypothesis $\mu=0$ is only used to assure that the sequence of the cocycled tableaux is defined. It means that this lemma is still true for the sequence $\left(T_{n+a}\right)_{n \geq 0}$. Thus we can define by induction as in proof of Proposition 4.2.2 an infinite sequence of tableaux $\left(T_{v_{j}}\right)_{j \geq 0}$ such that $T_{j_{0}}=T_{n+a}$ and for any $j, T_{v_{j+1}}$ has one column less than $T_{v_{j}}$. We derive a contradiction since the number of columns of $T_{a}$ is finite. It means that the sequence $\left(T_{n}\right)$ is finite.

Finally $T_{u}$ the last tableau of this sequence is necessarily a column such that $\hat{T}_{u}=T_{u}$ that is, $T_{u}$ is a column of weight 0 .

Example 4.3.2 The cocyclage operation is not authorized for $T=$\begin{tabular}{|l|l|}
$\overline{3}$ & $\overline{3}$ \\
\hline$\overline{2}$ & $\overline{1}$ \\
\hline 1
\end{tabular} . We have $\hat{T}=$

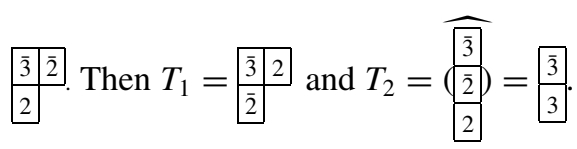

\subsection{Embedding of cyclage graphs}

Each connected component $\Gamma(T)$ contains a tableau $Y$ which admits no cocyclage. This tableau is necessarily unique. Suppose that $Y \in \mathbf{S T}(m)$.Then $\Gamma(T) \subset \mathbf{S T}(m)$ thus is finite. Moreover for any $Z \in \Gamma(T)$ there exists an integer $k$ such that $U^{(k)}(Z)=T$. This means that $\Gamma(T)$ has a tree structure.

Proposition 4.4.1 Let $\mu \in P_{n}$ and consider $T_{\mu} \in \mathbf{S T}(\mu)$. Suppose that there exists $j \leq i \leq n$ such that $\mu_{\bar{i}}>\mu_{\bar{j}} \geq 0$. Set $v \in P_{n}$ defined by $v_{\bar{k}}=\mu_{\bar{k}}$ for $k \neq i, j$, $v_{\bar{i}}=\mu_{\bar{i}}-1$ and $v_{\bar{j}}=\mu_{\bar{j}}+1$. Then there exists a tableau $T_{v} \in \mathbf{S T}(v)$ and a unique embedding from $\Gamma\left(T_{\mu}\right)$ to $\Gamma\left(T_{\nu}\right)$ which commutes with $U$ and preserves the shape of the tableaux. 
Proof: We have seen that $\Gamma\left(T_{\mu}\right)$ has a finite number of vertices. Write $m \geq n$ for the lowest integer such that $\Gamma\left(T_{\mu}\right)$ is contained in $\mathbf{S T}(m)$. By abuse of notation we also denote $\mu$ and $v$ the weights of $P_{m}$ defined by $\mu=\left(0, \ldots, 0, \mu_{\bar{n}}, \ldots, \mu_{\overline{1}}\right)$ and $v=\left(0, \ldots, 0, v_{\bar{n}}, \ldots, v_{\overline{1}}\right)$. Let $\sigma \in W_{m}$ such that $\sigma(\bar{i})=\bar{m}, \sigma(\bar{j})=\overline{m-1}$ and $\sigma(\bar{k})=\bar{k}$ for $k \neq i$, j. Set $\sigma(\mu)=\mu^{\prime}$ and $\sigma(v)=v^{\prime}$. Write $T_{\mu^{\prime}}=\sigma\left(T_{\mu}\right)$. We have $\Gamma\left(T_{\mu^{\prime}}\right) \subset \mathbf{S T}(m)$. Then for any $T \in \Gamma\left(T_{\mu^{\prime}}\right)$, $\tilde{f}_{m-1}(w(T)) \neq 0$. Indeed the crystal graph $G_{m}^{(m-1)}$ obtained by erasing in $G_{m}$ all the arrows of color $i \neq m-1$ and all the letters $x \notin\{\bar{m}, \overline{m-1}, m-1, m\}$ is a $U_{q}\left(s l_{2}\right)_{m-1^{-}}$ crystal where $U_{q}\left(s l_{2}\right)_{m-1}$ is the sub-algebra of $U_{q}\left(s p_{2 m}\right)$ isomorphic to $U_{q}\left(s l_{2}\right)$ generated by $e_{m-1}, f_{m-1}$ and $t_{m-1}$. The vertex $w(T)_{m-1}$ of $G_{m}^{(m-1)}$ obtained from $w(T)$ is of weight $\left(\mu_{\bar{m}}^{\prime}, \mu_{\overline{m-1}}^{\prime}\right) \neq 0$. Since $\mu_{\bar{i}}>\mu_{\bar{j}} \geq 0$ we have $\mu_{\bar{m}}^{\prime}>\mu_{\overline{m-1}}^{\prime} \geq 0$. Thus $w(T)_{m-1}$ is a highest weight vertex and there is an arrow of color $m-1$ and length $\mu_{\bar{m}}^{\prime}-\mu_{\overline{m-1}}^{\prime}$ which starts from $w(T)$.

Now consider $T \in \Gamma\left(T_{\mu^{\prime}}\right)$ such that $U(T)=T^{\prime}$ is defined. Write $w(T)=x_{*} w\left(T_{*}\right)$. We must have $x_{*} \neq \bar{m}$ since the cocyclage operation is authorized for $T$. Moreover $x_{*} \neq m$. Otherwise the first column of $T^{\prime}$ would contain the letters $m$ and $\bar{m}$ (because $\mu_{\bar{m}}^{\prime}>0$ ) and $T^{\prime} \notin \mathbf{S T}(m)$. We are going to prove that

$$
\tilde{f}_{m-1}\left(x_{*} w\left(T_{*}\right)\right)=x_{*} \tilde{f}_{m-1}\left(w\left(T_{*}\right)\right) .
$$

It suffices to establish (19) for $x_{*}=m-1$. When $x_{*}=m-1$ there is no letter $\overline{m-1}$ in the second row of $T$ since $T^{\prime} \in \mathbf{S T}(m)$. Thus all the letters $\overline{m-1}$ of $T$ belong to its first row. Denote by $T_{1}$ the sub tableau of $T$ containing all the columns whose the lowest letter is $\bar{m}$. The tableau $T$ can be regarded as the juxtaposition $T_{1} T_{2}$ of the tableaux $T_{1}$ and $T_{2}$ where $T_{2}$ is the sub-tableau obtained by considering the columns of $T$ which do not occur in $T_{1}$. Then $T_{1}$ do not contain any letter $m$ or $\overline{m-1}$ and $T_{2}$ do not contain any letter $\bar{m}$. Suppose that $\tilde{f}_{m-1}\left(x_{*} w\left(T_{*}\right)\right)=\tilde{f}_{m-1}\left(x_{*}\right) w\left(T_{*}\right)$. Then with the notation of Note 2.2.2 we can write $\rho\left(w\left(T_{2}\right)\right)=(+)^{s}$ since $x_{*}=m-1=+$ is not ignored during the encoding procedure. The pairs $( \pm)$ ignored are pairs $(m-1, \overline{m-1})$ or $(m-1, m)$ for $\bar{m}$ do not belong to $w\left(T_{2}\right)$. Since $\rho\left(w\left(T_{2}\right)\right)$ contains only symbols + , all the letters $\overline{m-1}$ can be paired with letters $m-1$. Thus the number of letters $m-1$ in $w\left(T_{2}\right)$ is strictly greater than that of letters $\overline{m-1}$. It is also true for $w(T)$ because $w\left(T_{1}\right)$ does not contain any letter $\overline{m-1}$. This contradicts the inequality $\mu_{m-1}^{\prime} \geq 0$. Thus (19) is true.

Denote by $V$ the symplectic tableau of reading $\tilde{f}_{m-1}\left(w\left(T_{\mu^{\prime}}\right)\right)$. We are going to prove that $\Psi: \Gamma\left(T_{\mu^{\prime}}\right) \rightarrow \Gamma(V)$ defined by $\Psi(T)=S$ if and only if $w(S)=\tilde{f}_{m-1}(w(T))$ is an embedding which commutes with $U$ and preserves the shape of the tableaux. We have $\Psi(U(T))=P\left(\tilde{f}_{m-1}\left(w\left(T_{*}\right) x_{*}\right)\right)$. Suppose that there exists $p \in\{1, \ldots, m\}$ such that $v_{\bar{p}}^{\prime}$ is equal to $r$ the number of columns of $S$. Then since $U(T)$ is defined and $v_{\bar{k}}^{\prime}=\mu_{\bar{k}}^{\prime}$ for any $k \neq m, m-1$ we must have $p \in\{m, m-1\}$. If $\nu_{\bar{m}}^{\prime}=r$ then we have $\mu_{\bar{m}}^{\prime}=$ $r+1$ which is impossible for $T$ contains only $r$ columns. If $v_{m-1}^{\prime}=r$ then we obtain $\mu_{\bar{m}}^{\prime} \geq r$ since $\mu_{\overline{m-1}}^{\prime}=r-1$ and $\mu_{\bar{m}}^{\prime}>\mu_{\overline{m-1}}^{\prime}$. This contradicts the fact that the cocyclage operation is authorized for $T$. Hence the cocyclage operation is authorized for $S$ and we 
can write

$$
\begin{aligned}
U(S)= & U(\Psi(T))=P\left(\xi\left(\tilde{f}_{m-1}\left(x_{*} w\left(T_{*}\right)\right)\right)=P\left(\xi\left(x_{*}\left(\tilde{f}_{m-1}\left(w\left(T_{*}\right)\right)\right)\right)\right.\right. \\
& =P\left(\tilde{f}_{m-1}\left(w\left(T_{*}\right)\right) x_{*}\right) .
\end{aligned}
$$

Thus we have to show that

$$
\tilde{f}_{m-1}\left(w\left(T_{*}\right) x_{*}\right)=\tilde{f}_{m-1}\left(w\left(T_{*}\right)\right) x_{*} .
$$

By (4) it is equivalent to

$$
\varphi_{m-1}\left(w\left(T_{*}\right)\right)>\varepsilon_{m-1}\left(x_{*}\right) .
$$

We have seen that $x_{*} \neq m$ and for $x_{*} \neq \overline{m-1}(20)$ is true since $\varepsilon_{m-1}\left(x_{*}\right)=0$ and $\varphi_{m-1}\left(w\left(T_{*}\right)\right) \geq 1$. Suppose that $x_{*}=\overline{m-1}$. Then the vertex of $G_{m}^{(m-1)}$ obtained from $w\left(T_{*}\right)$ as above is of weight $\left(\mu_{\bar{m}}, \mu_{\overline{m-1}}-1\right)$. Thus $\varphi_{m-1}\left(w\left(T_{*}\right)\right) \geq \mu_{\bar{m}}-\mu_{\overline{m-1}}+1 \geq 2$ for $\mu_{\bar{m}}>\mu_{\overline{m-1}}$. So (20) is satisfied. It is clear that $T$ and $\Psi(T)$ have the same shape. Moreover by (iii) of Lemma 4.2.1 $\Psi$ is the unique map from $\Psi \Gamma\left(T_{\mu^{\prime}}\right)$ to $\Gamma(V)$ which commutes with $U$ and preserves the shape of the tableaux. Finally, using $\sigma^{-1}$ we obtain from $\Psi$ a unique embedding $\Psi_{\sigma}$ satisfying $\Psi_{\sigma}(T)=\sigma^{-1} \Psi \sigma(T)$ from $\Gamma\left(T_{\mu}\right)$ to $\Gamma\left(T_{\nu}\right)$ with $T_{v}=\sigma^{-1} \Psi \sigma\left(T_{\mu}\right)$

Note that $T_{v}$ is not unique in general since $\Gamma(v)=\{\Gamma(T), T \in \mathbf{S T}(\mu)\}$ may contain fewer connected components isomorphic to $\Gamma\left(T_{v}\right)$.

Corollary 4.4.2 Let $\mu \in P_{n}^{+}$and $T_{\mu} \in \mathbf{S T}(\mu)$. Write respectively $m$ and $p$ for the sum of the non zero parts and the number of zero parts in $\mu$. Define $\kappa \in P_{m+p}^{+}$by $\kappa_{\bar{i}}=1$ for $p+1 \leq i \leq m+p$ and $\kappa_{i}^{\bar{i}}=0$ otherwise. Then there exists a tableau $T_{\kappa} \in \mathbf{S T}(\kappa)$ and $a$ unique embedding of $\Gamma\left(T_{\mu}\right)$ into $\Gamma\left(T_{\kappa}\right)$ which commutes with $U$ and preserves the shape of the tableaux.

Proof: The corollary directly follows by composing embeddings obtained in the previous Proposition.

Example 4.4.3 If $n=3$ and $\mu=(2,1,0)$ then $\kappa=(1,1,1,0)$. The cyclage graph $\left.\begin{array}{ll|l|l|l|l}\Gamma & \overline{3} & \overline{3} & \overline{2} & \overline{1} & 1\end{array}\right)$ of (18) may be uniquely embedded in $\Gamma\left(\begin{array}{l|l|l|l|l|l|l|l|l|l|l|l}\overline{4} & \overline{3} & \overline{2} & \overline{1} & 1\end{array}\right)$. 


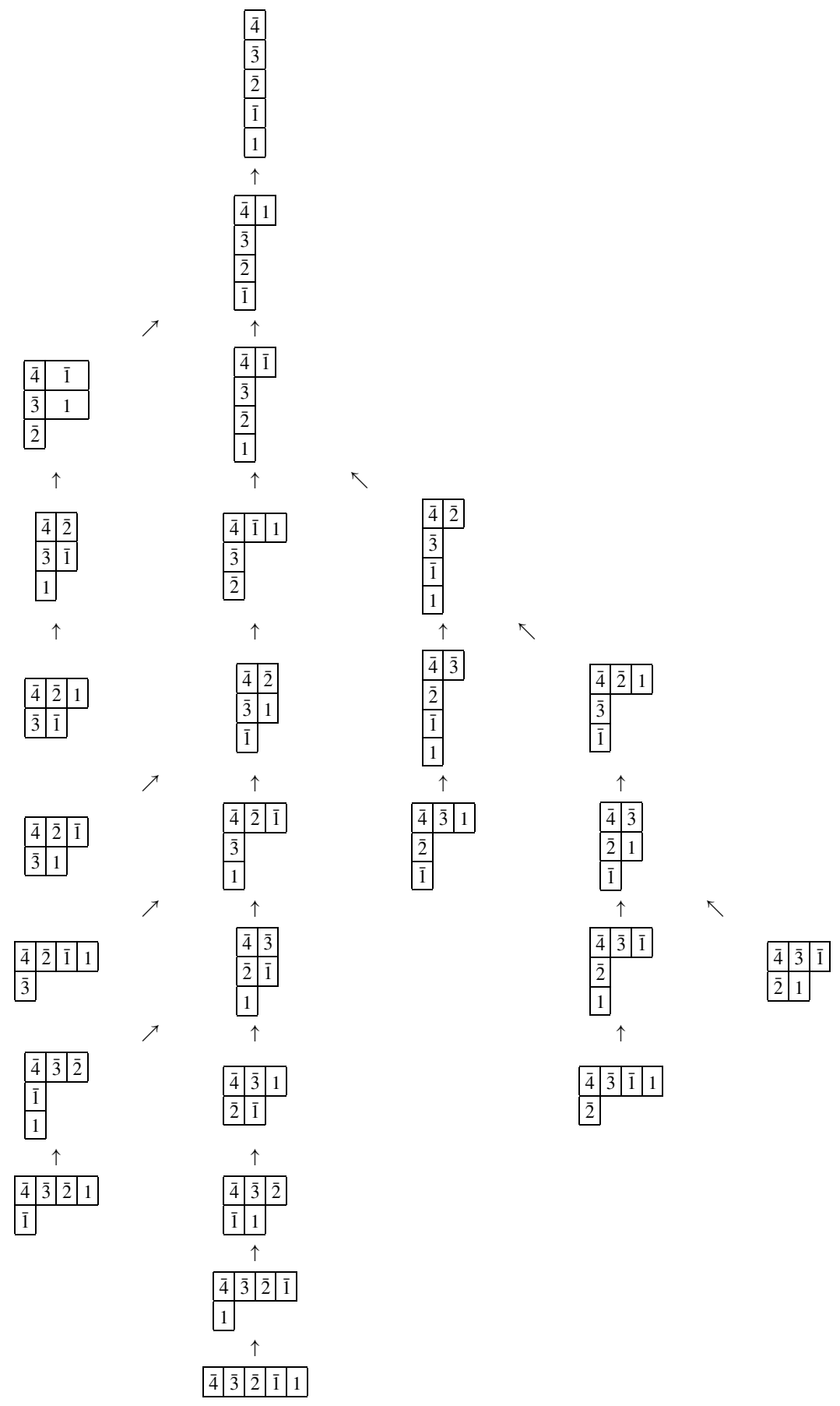




\section{A charge for symplectic tableaux}

\subsection{Definition of $c h_{n}$}

Definition 5.1.1 Let $C$ be a column of weight 0 . Write $E_{C}=\{i \geq 1, i \in C, i+1 \notin C\}$. The charge $\operatorname{ch}_{n}(C)$ of the column $C$ is

$$
\operatorname{ch}_{n}(C)=2 \sum_{i \in E_{C}}(n-i)
$$

Note that for any column $T$ of weight 0 ,

$$
\operatorname{ch}_{n+1}(C)=\operatorname{ch}_{n}(C)+2 \operatorname{card}\left(E_{C}\right) \quad \text { and } \quad \operatorname{ch}_{n+1}(t(C))=\operatorname{ch}_{n}(C)
$$

Moreover for any $i \geq 1$, we have $\varepsilon_{i}(w((C)))=\left\{\begin{array}{ll}1 & \text { if } i \in E_{C} \\ 0 & \text { otherwise }\end{array}\right.$ (see (5)). Thus for any $n$ admissible column $C$ of weight 0

$$
\operatorname{ch}_{n}(C)=2 \sum_{i=1}^{n-1}(n-i) \varepsilon_{i}(w(C)) .
$$

Now consider $T \in \mathbf{S T}(\mu)$ with $\mu \in P_{n}^{+}$. Let $\left\{T_{0}, \ldots, T_{p}\right\}$ with $T_{p}=C_{T}$ a column of weight 0 be the sequence obtained from $T$ as in Proposition 4.3.1).

Definition 5.1.2 The charge $\operatorname{ch}_{n}(T)$ is

$$
\operatorname{ch}_{n}(T)=\operatorname{ch}_{n}\left(C_{T}\right)+p .
$$

\section{Remark}

(i) For any tableau $T$ we have by Lemma 4.2.1 (ii)

$$
\operatorname{ch}_{n+1}(t(T))=\operatorname{ch}_{n}(T) \text {. }
$$

(ii) If the cocyclage operation is authorized in $T$, then $\operatorname{ch}_{n}(U(T))=\operatorname{ch}_{n}(T)-1$. Otherwise $\operatorname{ch}_{n}(\hat{T})=\operatorname{ch}_{n}(T)$.

\subsection{Conjectures}

Conjecture 5.2.1 Let $\Lambda_{k}^{A}$ and $\Lambda_{n-k}^{A}$ be respectively the $k$-th and $(n-k)$-th fundamental weights of $U_{q}\left(s l_{n}\right)$. Set $\lambda_{k}=\Lambda_{k}^{A}+\Lambda_{n-k}^{A}$. Then we have the equality:

$$
K_{\Lambda_{2 k}, 0}(q)=K_{\lambda_{k}, 0}^{A}\left(q^{2}\right)
$$


where $K_{\lambda_{k}, 0}^{A}\left(q^{2}\right)$ is the Kostka-Foulkes polynomial for the root system $A_{n-1}$ corresponding to $\mu=0$ evaluated at $q^{2}$.

Write $B(2 k)_{0}=\left\{b \in B\left(\Lambda_{2 k}\right)\right.$, wt $\left.(b)=0\right\}$. By identifying $U_{q}\left(s l_{n}\right)$ with the subalgebra of $U_{q}\left(s p_{2 n}\right)$ generated by the Chevalley's generators $e_{i}, f_{i}$ and $t_{i}, i=1, \ldots, n-1, B\left(\Lambda_{2 k}\right)$ has a structure of crystal graph for $U_{q}\left(s l_{n}\right)$ obtained by erasing all the arrows of color 0 that we denote $B^{A}\left(\Lambda_{2 k}\right)$. This graph decomposes into non isomorphic connected components and in this decomposition $B_{0}(2 k)$ is exactly the set of vertices of weight 0 of the connected component isomorphic to $B^{A}\left(\lambda_{k}\right)$

By Theorem 5.1 of [12] we can write

$$
K_{\lambda_{k}, 0}^{A}\left(q^{2}\right)=\sum_{w(C) \in B(2 k)_{0}} q^{2 \mathrm{~d}^{\prime}(w(C))}
$$

with $\mathrm{d}^{\prime}(w(C))=\sum_{i=1}^{n-1}(n-i) \varepsilon_{i}(w(C))$. Then it follows from (21) that Conjecture 5.2.1 is equivalent to the equality

$$
K_{\Lambda_{2 k}, 0}(q)=\sum_{w(C) \in B(2 k)_{0}} q^{\mathrm{ch}_{n}(C)}
$$

So by Corollary 3.2.4, this conjecture is true for $k=1$.

More generally many computations suggest that $\mathrm{ch}_{n}$ is an analogue for the root system $C_{n}$ of Lascoux-Schützenberger's charge on semi-standard tableaux.

Conjecture 5.2.2 Consider $\lambda, \mu \in P_{n}^{+}$. Then

$$
K_{\lambda, \mu}(q)=\sum_{w(T) \in B(\lambda)_{\mu}} q^{\mathrm{ch}_{n}(T)}
$$

where $B(\lambda)_{\mu}=\{T \in B(\lambda), \operatorname{wt}(T)=\mu\}$.

\section{Example 5.2.3}

1. Suppose $n=4, \lambda^{(1)}=(2,1,1,1)$ and $\mu^{(1)}=(1,1,1,0)$. There are 4 tableaux in $\mathbf{S T}(4)$ of shape $\lambda^{(1)}$ and weight $\mu^{(1)}$. They appear in the cyclage graph of Example 4.4.3. Set

$$
C=\left(\begin{array}{l}
\overline{4} \\
\overline{\overline{3}} \\
\overline{\overline{2}} \\
\hline \overline{1} \\
\hline 1
\end{array}\right) \text {. }
$$


We have $\operatorname{ch}_{4}(C)=0$ since $\widehat{C}=\frac{\overline{4}}{4}$. Hence the charges of these 4 tableaux are $1,2,3$ and 4. This gives the Kostka-Foulkes polynomial $K_{\lambda^{(1)}, \mu^{(1)}}(q)=q+q^{2}+q^{3}+q^{4}$.

2. Suppose $n=3, \lambda^{(2)}=(2,2,0)$ and $\mu^{(2)}=(0,0,0)$. There are 6 tableaux in ST(3) of shape $\lambda^{(2)}$ and weight $\mu^{(2)}$ :

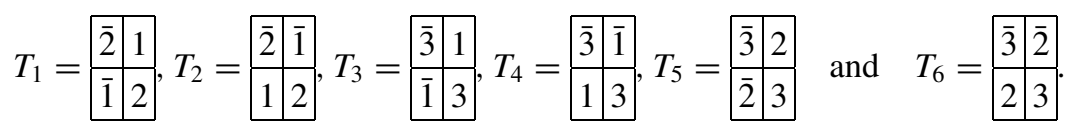

Note that $T_{2}, T_{3} \in \Gamma\left(\begin{array}{l|l|l|l|}\hline \overline{1} & \overline{1} & 1 & 1\end{array}\right)$ and $\Gamma\left(T_{1}\right)$ is given in (18). We obtain

$$
\operatorname{ch}_{3}\left(T_{1}\right)=2+\operatorname{ch}_{3}\left(\begin{array}{l}
\overline{2} \\
\overline{1} \\
\hline 1 \\
2
\end{array}\right)=4, \operatorname{ch}_{3}\left(T_{2}\right)=4+\operatorname{ch}_{3}\left(\begin{array}{l}
\overline{3} \\
\overline{1} \\
\hdashline \\
\hline 3
\end{array}\right)=8
$$

and $\operatorname{ch}_{3}\left(T_{3}\right)=6$. Moreover $T_{5}=t\left(T_{1}\right)$ and $T_{6}=t\left(T_{2}\right)$. Thus $\operatorname{ch}_{3}\left(T_{5}\right)=4-2=2$ and $\operatorname{ch}_{3}\left(T_{6}\right)=8-2 \times 2=4$. By an easy computation we obtain

$$
U^{(4)}\left(T_{4}\right)=\left(\begin{array}{l}
\overline{4} \\
\overline{1} \\
\hline 1 \\
\hline 4
\end{array}\right)
$$

Hence $\mathrm{ch}_{3}\left(T_{4}\right)=4+2(2-1)=6$. This gives the Kostka-Foulkes polynomial $K_{\lambda^{(2)}, \mu^{(2)}}$ $(q)=q^{2}+2 q^{4}+2 q^{6}+q^{8}$.

\section{Remark}

(i) Once $\mathrm{ch}_{n}$ defined on $\mathbf{S T}$, it is possible to define $\mathrm{ch}_{n}$ for any words of $\mathcal{C}_{\infty}^{*}$ by setting

$$
\operatorname{ch}_{n}(w)=\operatorname{ch}_{n}(P(w))
$$

Then given $w_{1}, w_{2} \in \mathcal{C}_{n}^{*}$, the congruence $w_{1} \equiv w_{2}$ implies that $\operatorname{ch}_{n}\left(w_{1}\right)=\operatorname{ch}_{n}\left(w_{2}\right)$, that is $\mathrm{ch}_{n}$ is a plactic invariant. We recover a property of the Lascoux-Schützenberger's charge $\mathrm{ch}_{A}$ for type $A$ [14] [20]. Nevertheless, it seems difficult to define $\mathrm{ch}_{n}$ directly 
on words as it is possible for $\mathrm{ch}_{A}$. In [12], the statistic $\mathrm{ch}_{A}$ is characterized in terms of the combinatorics of crystal graphs. We have not found such a characterization for the symplectic charge $\mathrm{ch}_{n}$.

(ii) It seems to be impossible to define a simple charge statistic on $\mathbf{S T}(n)$ by using a cocyclage operation taking into account the contraction relation (9) and relevant for

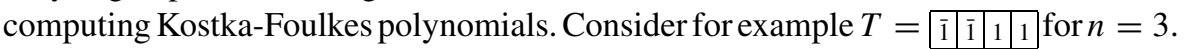
If we apply cocyclages operations based on the complete insertion scheme (with the contraction relations) we obtain the symplectic tableaux of $\mathbf{S T}(3)$,

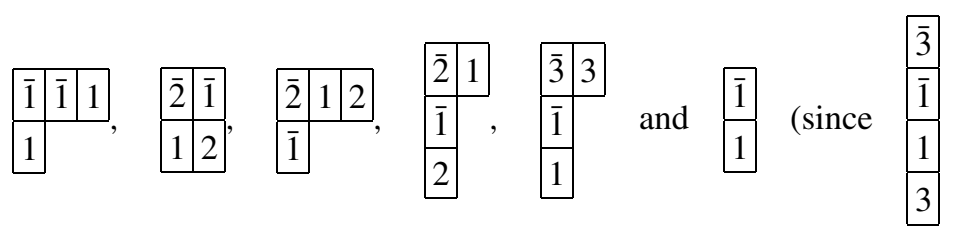

is not a 3-admissible column). We know by Proposition 3.2.3 that a charge for $T$ must necessarily be odd and by Corollary 3.2.4 a charge for $\frac{1}{1}$ must be even. So we can not deduce the charge of $T$ from that of $\frac{1}{1}$ by simply counting the number of cocyclage
operations.

(iii) Lascoux-Schützenberger's proof of the equality

$$
K_{\lambda, \mu}(q)=\sum_{w(T) \in B(\lambda)_{\mu}} q^{\mathrm{ch}_{A}(T)}
$$

for type $A$ is based on the Morris recurrence formula. We have seen that Theorem 3.2.1 can be regarded as an analogue of this formula for type $C_{n}$. It permits to decompose a Kostka-Foulkes polynomial for type $C_{n}$ in terms of Kostka-Foulkes polynomials for type $C_{n-1}$. Unfortunately a charge statistic must take into account the contraction relations to be compatible with the decomposition obtained in this way since the partitions $\lambda$ such that $B(\lambda)$ appears in a decomposition of type $B(\gamma) \otimes B\left((r)_{n-1}\right)$ may be such that $|\lambda|<|\mu|$. This is a reason why we are not able to deduce Conjecture 5.2.2 from Theorem 3.2.1.

\section{References}

1. R.-K. Brylinski, "Limits of weight spaces, Lusztig's $q$-analogs and fiberings of adjoint orbits," J. Amer. Math. Soc. 2(3) (1989), 517-533.

2. P. Caldero, "On harmonic elements for semi-simple Lie algebras," Advances in Mathematics 166, (2002), 73-99.

3. V. Chari and A. Presley, A Guide to Quantum Groups, Cambridge University Press, 1994.

4. J. Hong and S.J. Kang, Introduction to Quantum Groups and Crystals Bases, A.M.S 2002, GSM/12.

5. J.C. Jantzen, "Lectures on quantum groups," Graduate Studies in Math. 6 (A.M.S 1995).

6. A. Joseph, G. Letzer, and S. Zelikson, "On the Brylinski-Kostant filtration," J. Amer. Math. Soc. 13(4) (2000), 945-970. 
7. M. Kashiwara, "Crystallizing the $q$-analogue of universal enveloping algebra," Commun. Math. Phys. 133 (1990), 249-260.

8. M. Kashiwara, "On crystal bases of the $q$-analogue of universal enveloping algebras," Duke Math. J., 63 (1991), 465-516.

9. M. Kashiwara, “Crystallization of quantized universal enveloping algebras,” Sugaku Expositiones 7 (1994), 99-115

10. M. Kashiwara, "On crystal bases," Canadian Mathematical Society, Conference Proceedings 16 (1995) 155197.

11. M. Kashiwara and T. Nakashima, "Crystal graphs for representations of the $q$-analogue of classical Lie algebras," Journal of Algebra 165 (1994), 295-345.

12. A. Lascoux, B. Leclerc, and J-Y. Thibon, "Crystal graphs and $q$-analogue of weight multiplicities for the root system $A_{n}$, , Letters in Mathematical Physics 35 (1995), 359-374.

13. A. Lascoux and M.-P. Schützenberger, "Le monoïde plaxique," in A. de Luca (Ed.), Non Commutative Structures in Algebra and Geometric Combinatorics, Quaderni della Ricerca Scientifica del C.N.R., Roma, 1981.

14. A. Lascoux and M.-P. Schützenberger, "Sur une conjecture de H.O Foulkes," CR Acad Sci Paris 288 (1979), 95-98.

15. C. Lecouvey, "Schensted-type correspondence, Plactic Monoid and Jeu de Taquin for type $C_{n}$," J. Algebra 247 (2002), 295-331.

16. M. Lothaire, "Encyclopedia of mathematics and its applications," Algebraic Combinatorics of Words, 90 , 164-196.

17. G. Lusztig, "Singularities, character formulas, and a $q$-analog of weight multiplicities, Analyse et topologie sur les espaces singuliers (II-III)," Asterisque 101/102 (1983), 208-227.

18. I.G. Macdonald, Symmetric Functions and Hall Polynomials, 2nd ed., Oxford Mathematical Monograph, Oxford University Press, New York, 1995.

19. K. Nelsen and A. Ram, Kostka-Foulkes Polynomials and Macdonald Spherical Functions in Surveys in Combinatorics, 2003 (Bangor), Lect. Notes London Math. Soc. 307, Cambridge Univ. Press, Cambridge, pp. 325-370

20. M-P. Schützenberger, "Propriétés nouvelles des tableaux de Young, Séminaire Delange-Pisot-Poitou," 19ème année 26 (1977/78). 